\title{
The chemistry of planetary nebulae and HII regions in the dwarf galaxies Sextans A and B from deep VLT spectra ${ }^{\star}$
}

\author{
L. Magrini ${ }^{1}$, P. Leisy ${ }^{2,3}$, R. L. M. Corradi ${ }^{3}$, M. Perinotto ${ }^{1}$, A. Mampaso ${ }^{2}$, and J. M. Vílchez \\ 1 Dipartimento di Astronomia e Scienza dello Spazio, Universitá di Firenze, L.go E. Fermi 2, 50125 Firenze, Italy \\ e-mail: laura@arcetri.astro.it \\ 2 Instituto de Astrofísica de Canarias, c. Vía Láctea s/n, 38200, La Laguna, Tenerife, Canarias, Spain \\ 3 Isaac Newton Group of Telescopes, Apartado de Correos 321, 38700 Santa Cruz de La Palma, Canarias, Spain \\ ${ }^{4}$ Instituto de Astrofísica de Andalucía (CSIC) Apartado de Correos 3004, 18080 Granada, Spain
}

Received 2 March 2005 / Accepted 25 July 2005

\section{ABSTRACT}

Spectroscopic observations obtained with the VLT of one planetary nebula (PN) in Sextans A and of five PNe in Sextans B and of several $\mathrm{H}$ II regions in these two dwarf irregular galaxies are presented. The extended spectral coverage, from 320.0 to $1000.0 \mathrm{~nm}$, and the large telescope aperture allowed us to detect a number of emission lines, covering more than one ionization stage for several elements (He, $\mathrm{O}$, $\mathrm{S}, \mathrm{Ar}$ ). The electron temperature diagnostic [O III] line at $436.3 \mathrm{~nm}$ was measured in all six PNe and in several $\mathrm{H}$ II regions allowing for an accurate determination of the ionic and total chemical abundances by means of the Ionization Correction Factors method. For the time being, these PNe are the farthest ones where such a direct measurement of the electron temperature is obtained. In addition, all PNe and $\mathrm{H}$ II regions were also modelled using the photoionization code CLOUDY (Ferland et al. 1998, PASP, 110, 761). The physico-chemical properties of PNe and $\mathrm{H}$ II regions are presented and discussed. A small dispersion in the oxygen abundance of $\mathrm{H}$ II regions was found in both galaxies: $12+\log (\mathrm{O} / \mathrm{H})=7.6 \pm 0.2$ in Sextans A, and $7.8 \pm 0.2$ in Sextans B. For the five PNe of Sextans B, we find that $12+\log (\mathrm{O} / \mathrm{H})=8.0 \pm 0.3$, with a mean abundance consistent with that of $\mathrm{H}$ II regions. The only PN known in Sextans A appears to have been produced by a quite massive progenitor, and has a significant nitrogen overabundance. In addition, its oxygen abundance is 0.4 dex larger than the mean abundance of $\mathrm{H}$ II regions, possibly indicating an efficient third dredge-up for massive, low-metallicity PN progenitors. The metal enrichment of both galaxies is analyzed using these new data.

Key words. ISM: abundances - ISM: planetary nebulae: individual: Sextans A Sextans B - ISM: HII regions galaxies: individual: Sextans A Sextans B - galaxies: Local Group - galaxies: abundances

\section{Introduction}

Sextans A and Sextans B are both dwarf irregular galaxies (Ir V and Ir IV-V morphological types, respectively, cf. van den Bergh 2000, hereafter vdB00) with approximately the same V luminosity and located at a very similar distance (1.3 Mpc, Dolphin et al. 2003 for Sextans A and vdB00 for Sextans B). Their separation in the sky is also relatively small ( $\sim 10$ degrees), which corresponds to about $280 \mathrm{kpc}$ at the adopted distance. Moreover their velocity difference is only $23 \pm 6 \mathrm{~km} \mathrm{~s}^{-1}$. All these properties suggest a common formation for these two galaxies, probably together with NGC 3109 and the Antlia galaxy, also at a similar distance and location in the sky. Considering the mean distance of the four galaxies from the barycentre of the Local Group (LG), 1.7 Mpc, this

* Based on observations obtained at the $8.2 \mathrm{~m}$ VLT telescope (Paranal, Chile) operated by ESO (Proposal 072.B-0395) and at the $4.2 \mathrm{~m}$ WHT telescope (La Palma, Spain) operated by the Isaac Newton Group in the Spanish Observatorio del Roque de Los Muchachos of the Instituto de Astrofisica de Canarias. sub-group is located beyond the zero velocity surface of the LG (cf. vdB00) and can be considered the nearest external group of galaxies. Then these galaxies are particularly interesting as they represent a group of dwarf galaxies relatively isolated from giant galaxies.

In dIr galaxies, star formation is generally active, as shown by the conspicuous number of $\mathrm{H}$ II regions that they contain. There are several photometric and spectroscopic studies of $\mathrm{H}$ II regions in both galaxies. Photometry of $\mathrm{H}$ II regions in Sextans A was obtained by Hodge (1974), Aparicio \& Rodríguez-Ulloa (1992), and Hodge et al. (1994), the latter one being the most complete survey with $25 \mathrm{H}$ II regions detected. Hunter et al. (1993) studied large ionized gas structures outside normal $\mathrm{H}$ II regions, like shells and filaments generated by the action of massive stars through winds and supernova explosions. Spectra of four $\mathrm{H}$ II regions were obtained by Skillman et al. (1989), from which an oxygen abundance was derived. The brightest H II regions of Sextans B were first catalogued by Hodge (1974). Strobel et al. (1991) classified twelve $\mathrm{H}$ II regions, whereas oxygen abundance was derived for four 
H II regions by Skillman et al. (1989). Note, however, that these spectroscopic studies were generally not deep enough (except for one or two objects) to allow direct measurement of the nebular electron temperature via detection of the [O III] $436.3 \mathrm{~nm}$ line, causing an important source of uncertainty in the determination of their chemical abundances.

Planetary nebulae (PNe) are known to be good tracers of intermediate-age stellar populations in galaxies. One PN was discovered in Sextans A by Jacoby \& Lesser (1981), and then reidentified by Magrini et al. (2003). In Sextans B, five PNe are known (Magrini et al. 2002). To date, no spectroscopic studies were done of these PNe.

As remarked by Mateo (1998) "No two LG dwarfs have the same star-formation history". This is true not only when comparing galaxies of different morphological type, luminosity, or mass, but also when comparing galaxies which are very similar in many aspects, as these two dwarf irregulars. In fact, in spite of a probable common origin, Sextans A and Sextans B show a different star formation history as indicated by the different amounts of stars in the various evolutionary phases, and also reflected in the different number of $\mathrm{PNe}$ and $\mathrm{H}$ II regions observed. Sextans A possesses a conspicuous old stellar population (10-14 Gyrs ago) and shows evidence for recent star formation starting about 1-2 Gyrs ago, whereas the amount of star formation at intermediate ages (2-10 Gyrs ago) was modest (see also Dolphin et al. 2003). On the other hand, Sextans B exhibits a very strong star formation at recent and intermediate ages (1-4 Gyrs ago), together with a very old population (cf. Mateo 1998). The different numbers of PNe, 5 in Sextans B vs. 1 in Sextans A, was interpreted by Magrini et al. (2002) to be consistent with the different star formation histories of the two galaxies for the age range covered by the PN progenitors, Sextans B showing a stronger star formation rate than Sextans A during the past 2-10 Gyrs. The different star formation history might imply a different chemistry. In this paper, we analyze the chemical content of Sextans A and Sextans B, presenting spectroscopic data of $\mathrm{PNe}$, whose progenitors belong to old and intermediate age populations, and $\mathrm{H}$ II regions, representative of the youngest population.

The paper is organized as follows. Section 2 describes the observations and the data reduction. Section 3 presents the chemical abundances obtained for $\mathrm{PNe}$ and $\mathrm{H}$ II regions, and discusses the implications on the properties and evolution of the galaxies. In Sect. 4 the notable case of the PN in Sextans A is examined. The summary is in Sect. 5.

\section{Observations and data reduction}

All PNe known in these galaxies (1 in Sextans A and 5 in Sextans B) and a sample of H II regions (9 and 8, respectively) have been observed in December 2003 with the $8.2 \mathrm{~m}$ VLT (ESO, Paranal) equipped with the FORS2 spectrograph in multi-object spectroscopy (MOS) observing mode. Pre-imaging, needed to create the MOS masks, was first obtained through an $\mathrm{H} \alpha$ filter (central wavelength $656.3 \mathrm{~nm}$, $F W H M 6.1 \mathrm{~nm})$ and a $R$ filter $(655 / 165.0 \mathrm{~nm})$. The exposure times for each galaxy were $2 \times 120 \mathrm{~s}$ in $\mathrm{H} \alpha$ and $2 \times 10 \mathrm{~s}$ in $R$. In the continuum-subtracted $\mathrm{H} \alpha-R$ frames, emission-line objects were identified and their position used to build two MOS masks, one per galaxy.

Spectroscopy was then secured during four consecutive nights (22-25 December). The seeing during the observations was 0 ' $^{\prime} 8$. The MOS mask used to observe Sextans A contained 32 slits and the one for Sextans B 28 slits. The slits had a width of $0.8^{\prime \prime}$, while their length varied from $3^{\prime \prime}$ to $25^{\prime \prime}$, according to the size of the object to be observed and to the crowding of the area of the galaxy. The slits corresponding to compact objects like PNe were extended enough to include also some adjacent sky spectrum. Several independent slits were used to obtain sky spectra to be subtracted from those of extended H II regions, which completely fill their slitlets. Spectra were obtained using FORS2 with the $300 \mathrm{~V}$ and 300I gratings, providing "blue" and "red" spectra with a total coverage from $\sim 320.0 \mathrm{~nm}$ to $\sim 1000.0 \mathrm{~nm}$ at a reciprocal dispersion of $0.3 \mathrm{~nm} \mathrm{pix}{ }^{-1}$ and an effective resolution of $0.6 \mathrm{~nm}$. For each galaxy, a total of eight exposures were taken: three with the $300 \mathrm{~V}$ grating (total exposure time $5400 \mathrm{~s}$ ), three using the $300 \mathrm{I}$ one $(3600 \mathrm{~s})$, and also two short exposures for each grating (60 s) to avoid saturation of the most intense emission lines, as $\mathrm{H} \alpha$ and [O III] $500.7 \mathrm{~nm}$. A spectrophotometric standard star, GD 108, was observed once in blue and red during two consecutive nights. The data were reduced using the IRAF LONGSLIT tasks and the MIDAS MOS and LONG packages.

\subsection{Images}

The $\mathrm{H} \alpha-R$ FORS 2 images of Sextans A and Sextans B are shown in Fig. 1. All PNe in Magrini et al. (2002, 2003) were re-identified, together with a number of known HII regions. Compared to previous studies (Hodge et al. 1994; Strobel et al. 1991), several new unresolved $\mathrm{H} \alpha$ emission-line objects were found in both galaxies. When comparing their coordinates with the images in Magrini et al. (2002, 2003), no associated [O III] emission was found, and therefore we do not consider them as new candidate PNe. We observed spectroscopically two of them, one in Sextans A and one in Sextans B, confirming that they are stars with a very strong $\mathrm{H} \alpha$ line in emission. A detailed analysis of these objects via colour-colour diagrams and spectroscopy will be presented in a forthcoming paper.

In Fig. 1, all PNe and $\mathrm{H}$ II regions observed spectroscopically are marked with their identification numbers as in Table 1 for $\mathrm{H}$ II regions and as in Magrini et al. $(2002,2003)$ for PNe.

\subsection{Spectra}

An important feature of this spectroscopic study is the homogeneous set of observational data and analysis method used to measure chemical abundances for both PNe and $\mathrm{H}$ II regions. Furthermore, the VLT allows us to obtain spectra which are deep enough to measure most basic diagnostic emission lines in spite of the significant distance of the galaxies. The electron temperature diagnostic [O III] 436.3 was measured in all $\mathrm{PNe}$, and in seven $\mathrm{H}$ II regions out of a sample of 17 . In few other H II regions (four in Sextans A and one in Sextans B), 

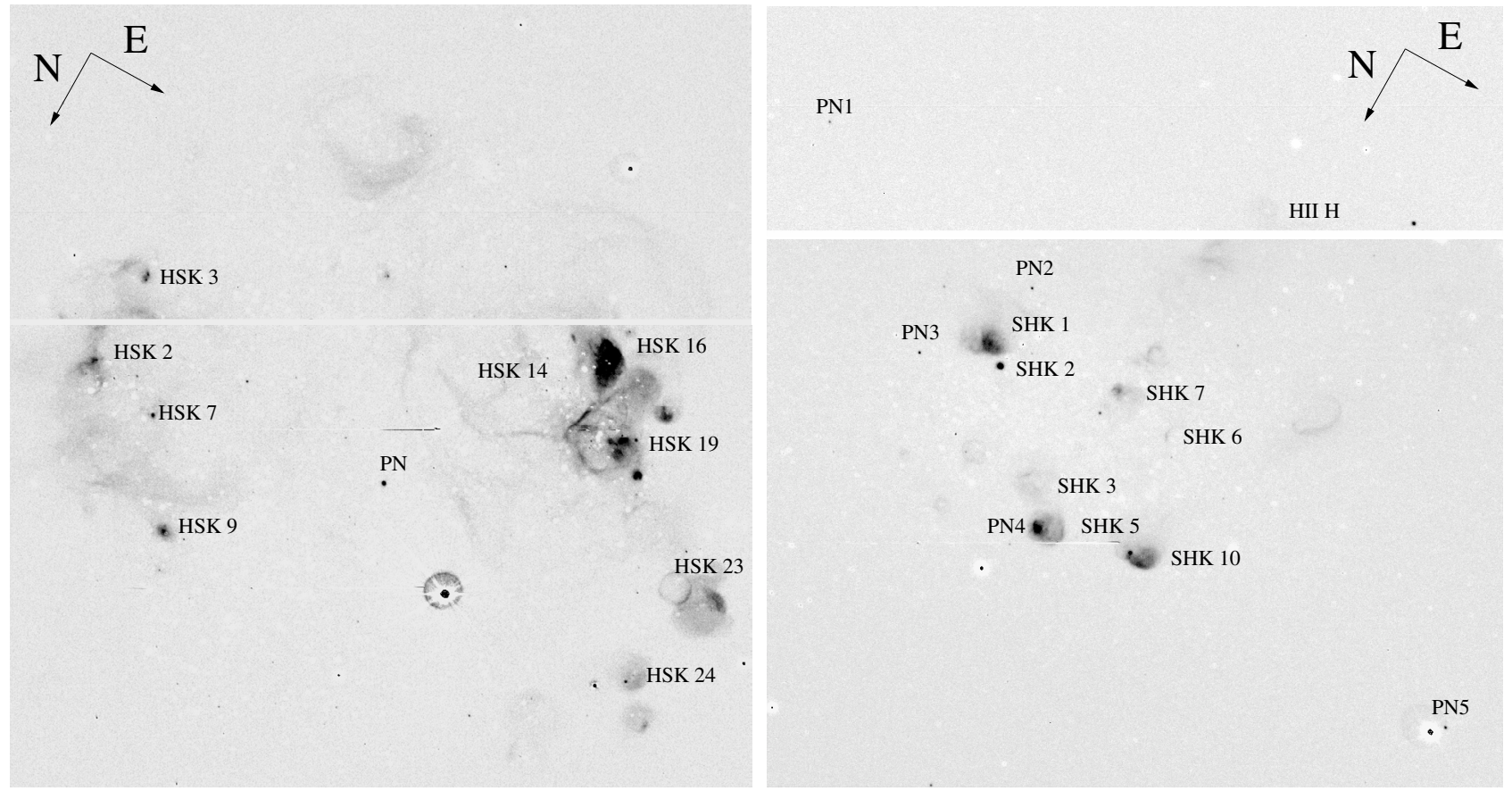

Fig. 1. VLT H $\alpha-R$ images of Sextans A (left $)$ and Sextans B (right). The f.o.v. is $2^{\prime} \times 2^{\prime}$. The H II regions and PNe observed spectroscopically are indicated with their identification numbers as in Table 1 for $\mathrm{H}$ II regions and as in Magrini et al. $(2002,2003)$ for PNe.

Table 1. The observed H II regions in Sextans A and Sextans B. The centres of the slits are reported. The adopted names of $\mathrm{H}$ II regions are from Hodge et al. (1994) for Sextans A and from Strobel et al. (1991) for Sextans B. The HII region not identified in previous works is labeled as $\mathrm{H} \mathrm{II} \mathrm{H}$.

\begin{tabular}{lll}
\hline \hline Name & RA & Dec \\
& \multicolumn{2}{c}{ J2000.0 } \\
\hline Sextans A & & \\
HKS2 & $10: 10: 53.6$ & $-4: 41: 16.9$ \\
HKS3 & $10: 10: 53.8$ & $-4: 41: 57.2$ \\
HKS7 & $10: 10: 55.4$ & $-4: 41: 11.0$ \\
HKS9 & $10: 10: 57.0$ & $-4: 40: 30.6$ \\
HKS14 & $10: 11: 04.1$ & $-4: 42: 49.3$ \\
HKS16 & $10: 11: 05.6$ & $-4: 42: 31.5$ \\
HKS19 & $10: 11: 06.9$ & $-4: 42: 17.8$ \\
HKS23 & $10: 11: 09.2$ & $-4: 40: 53.2$ \\
HKS24 & $10: 11: 09.6$ & $-4: 41: 36.3$ \\
\hline Sextans B & & \\
SHK1 & $9: 59: 57.7$ & $5: 19: 36.7$ \\
SHK2 & $9: 59: 58.3$ & $5: 19: 46.3$ \\
SHK3 & $9: 59: 59.9$ & $5: 20: 17.4$ \\
SHK5 & $10: 00: 00.4$ & $5: 20: 25.2$ \\
SHK7 & $10: 00: 00.8$ & $5: 19: 38.3$ \\
SHK6 & $10: 00: 02.2$ & $5: 19: 44.8$ \\
SHK10 & $10: 00: 03.1$ & $5: 20: 31.8$ \\
H II H & $10: 00: 05.3$ & $5: 17: 58.7$ \\
\hline
\end{tabular}

an upper limit to the flux of the [O III] 436.3 line could be derived. The broad spectral coverage, from 320.0 to $1000.0 \mathrm{~nm}$, allowed us the detection of many recombination $(\mathrm{H} \mathrm{I}$, He I, $\mathrm{He}$ II) and forbidden lines of several ions of $\mathrm{O}$ ([O I], [O II],
[O III]), S ([S II], [S III]), Ar ([Ar III], [Ar IV]), Ne ([Ne III]), and $\mathrm{N}$ ([N II]). Note that the [S III] lines at 906.9 and $953.2 \mathrm{~nm}$ are generally blended with saturated atmospheric absorption features. In this case, the heliocentric velocities of both galaxies, $324 \mathrm{~km} \mathrm{~s}^{-1}$ for Sextans A and $300 \mathrm{~km} \mathrm{~s}^{-1}$ for Sextans B, and the excellent sky transparency at these wavelength in Paranal, allowed us to use safely these lines to determine the $\mathrm{S}^{++}$abundances.

The spectra of the PNe are presented in Figs. 2 and 3, and those of H II regions in Figs. 4-7. Identification of some emission lines is labeled. Note the large number of emission lines detected with a good signal to noise ratio, and the relatively strong blue continuum emission visible in a number of $\mathrm{H}$ II regions, which is likely produced by the exciting stars.

It should be noted that although the observations were carried out at relatively low airmasses, the slits were not aligned exactly to the parallactic angle at the time of the observations: for Sextans A the mean difference between the parallactic angle and the position angle of the slits was $45^{\circ}$ and the mean airmass was 1.15 , while for Sextans B they were $35^{\circ}$ and 1.25 , respectively. This implies that, given the relatively narrow slit, some amount of light is lost especially in the blue end of the spectra due to differential atmospheric refraction. In order to estimate these light-losses for point sources like PNe, we have calculated the displacement, as a function of wavelength, of the centroid of an object from the midpoint of the slit along the direction perpendicular to its length, using the figures in Filippenko (1982). In doing so, we consider that sources are well centred at $\mathrm{H} \alpha$, which is the passband at which acquisition images were taken to centre objects in their slitlets for spectroscopy. We have then calculated the amount of light lost (relative to that at $\mathrm{H} \alpha$ ) because of these displacements from the 


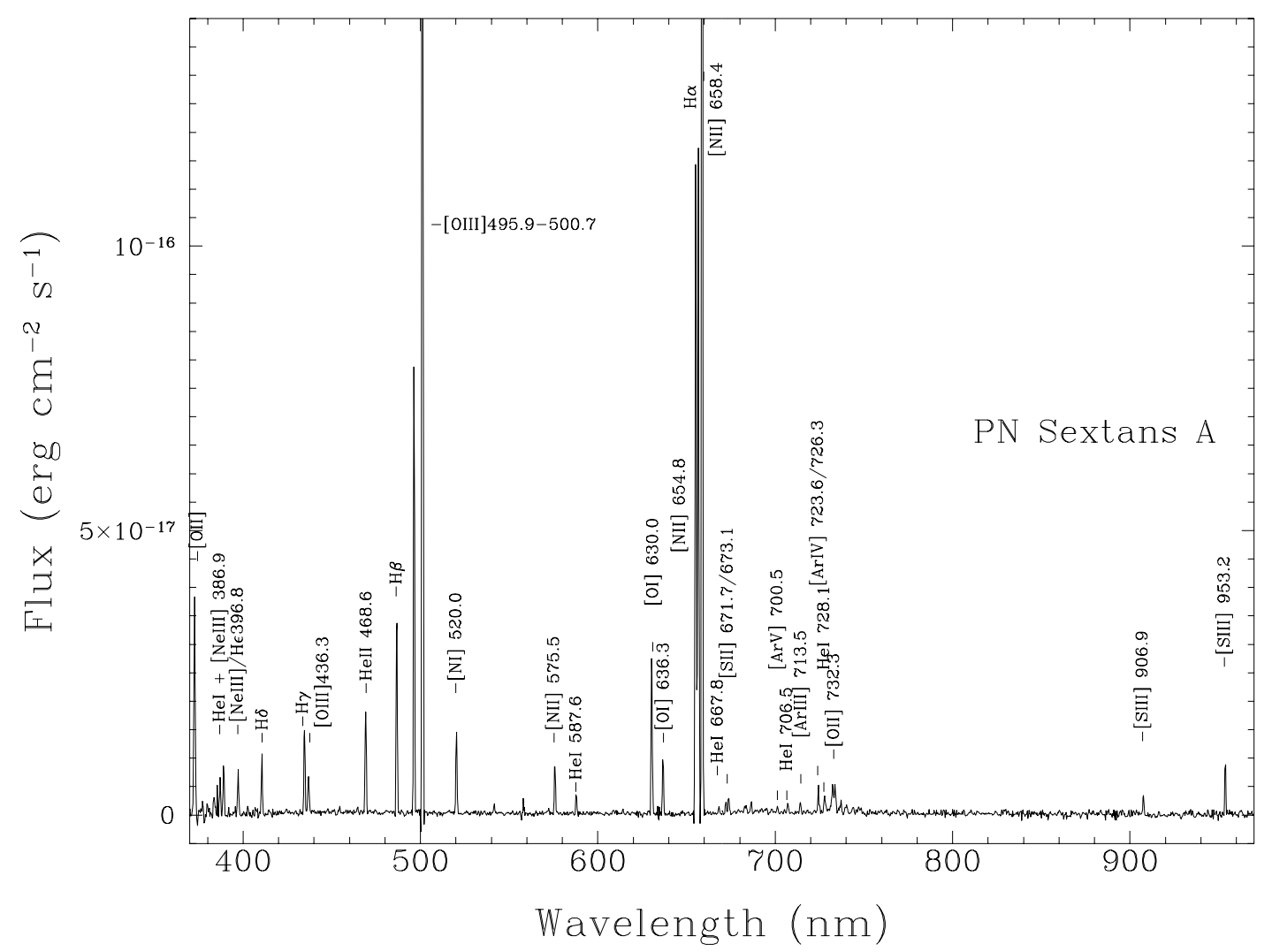

Fig. 2. VLT spectrum of the planetary nebula in Sextans A.

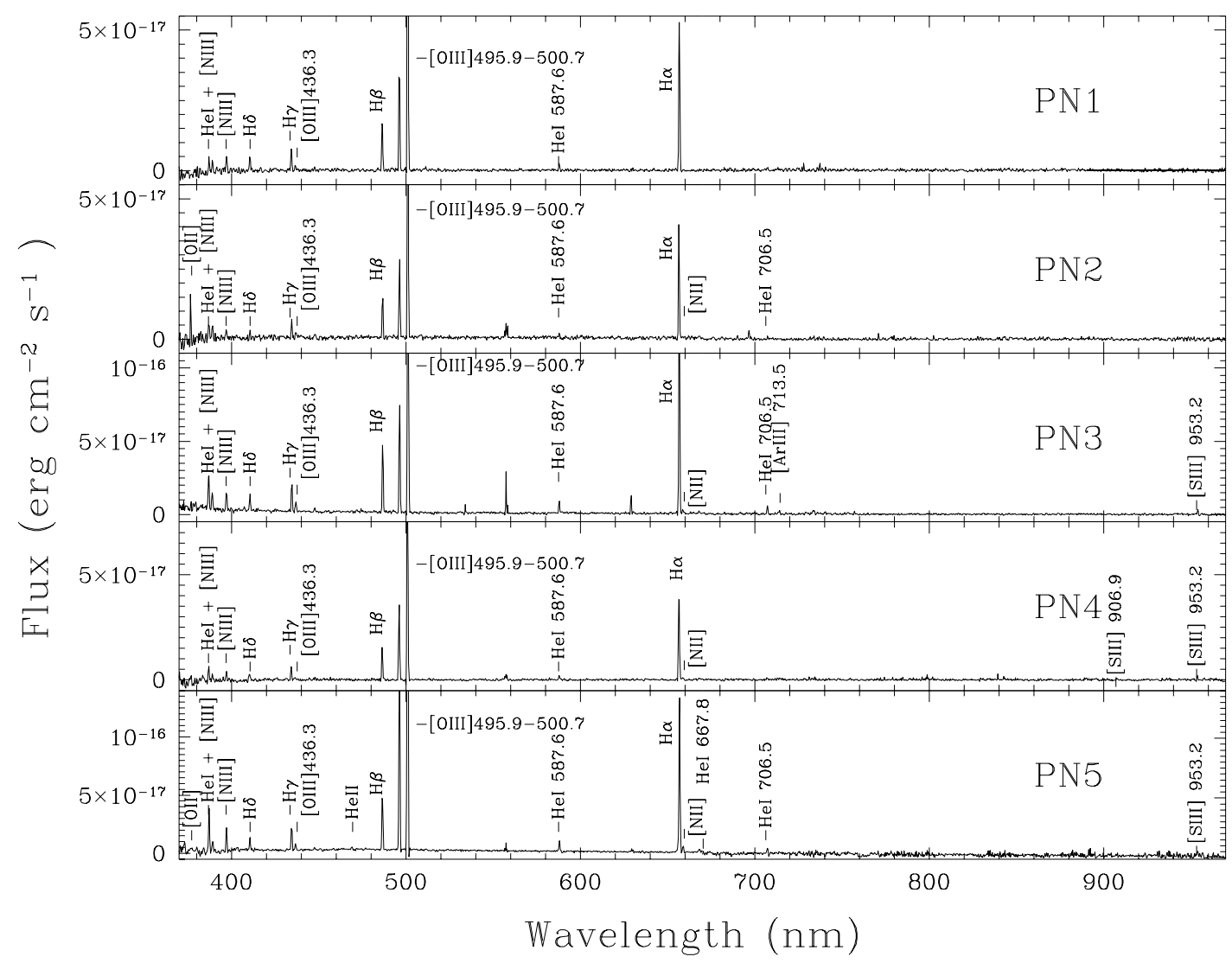

Fig. 3. VLT spectra of the five planetary nebulae in Sextans B. Identification numbers come from Magrini et al. (2002). 


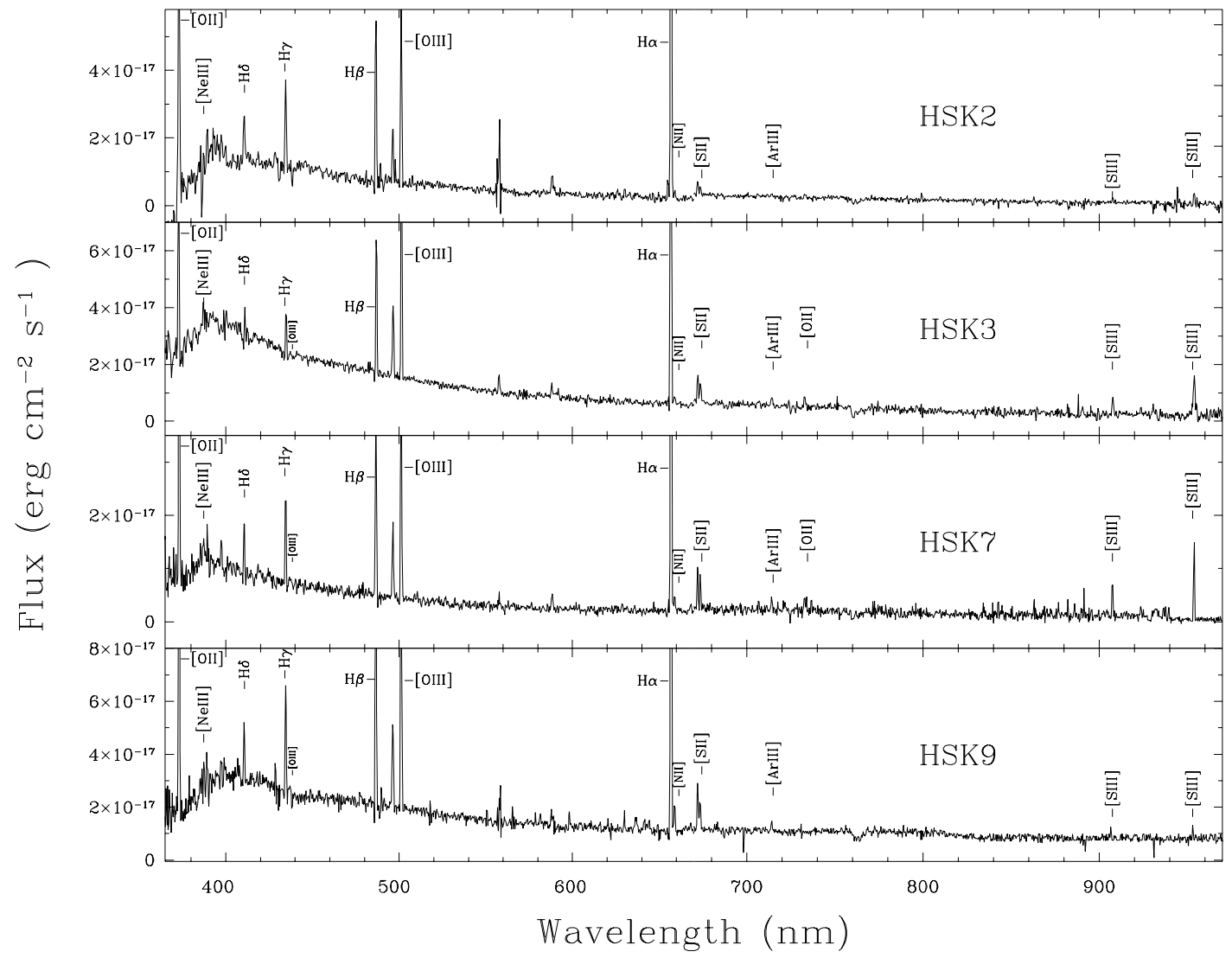

Fig. 4. VLT spectra of four H II regions in Sextans A.

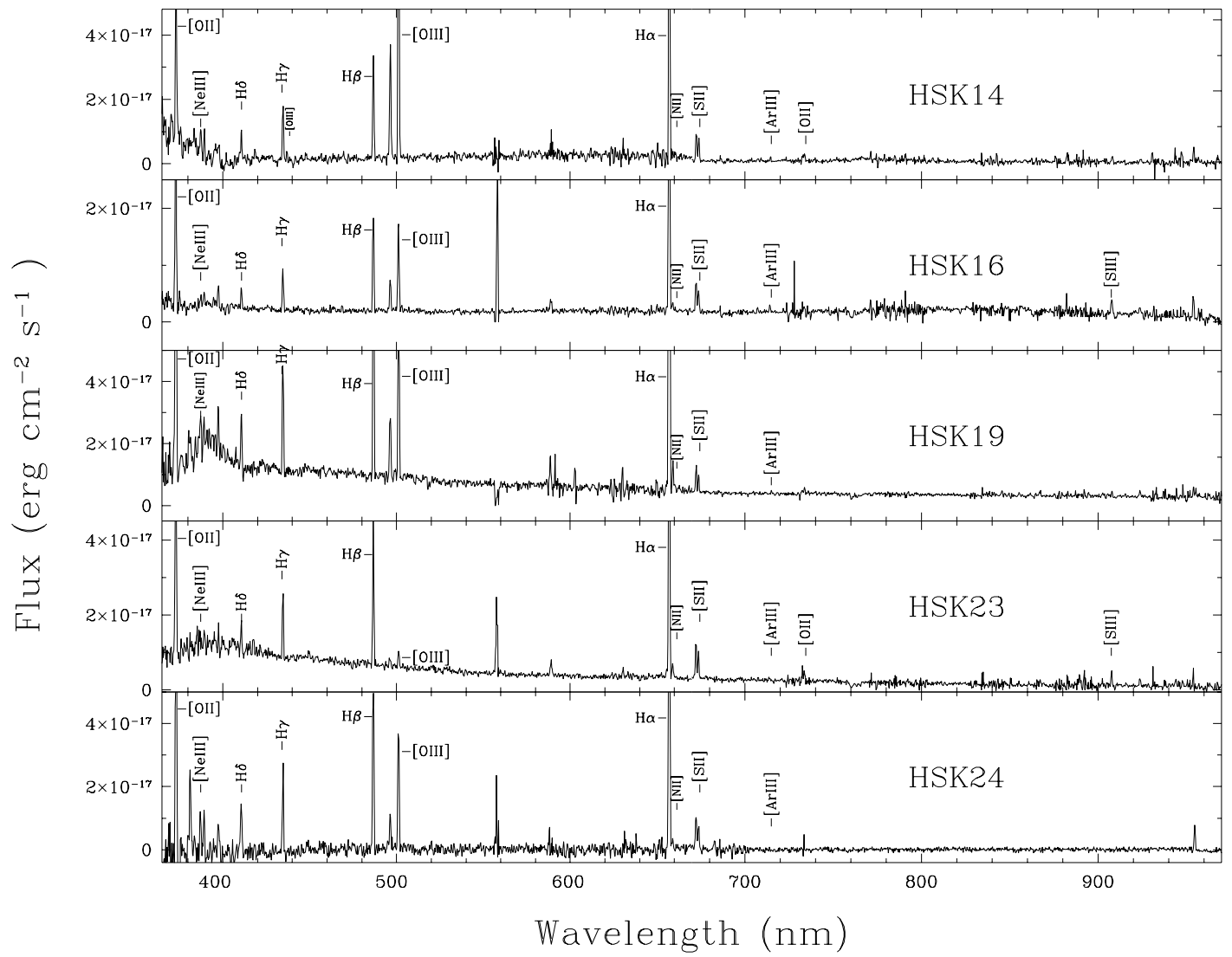

Fig. 5. VLT spectra of another five H II regions in Sextans A. 


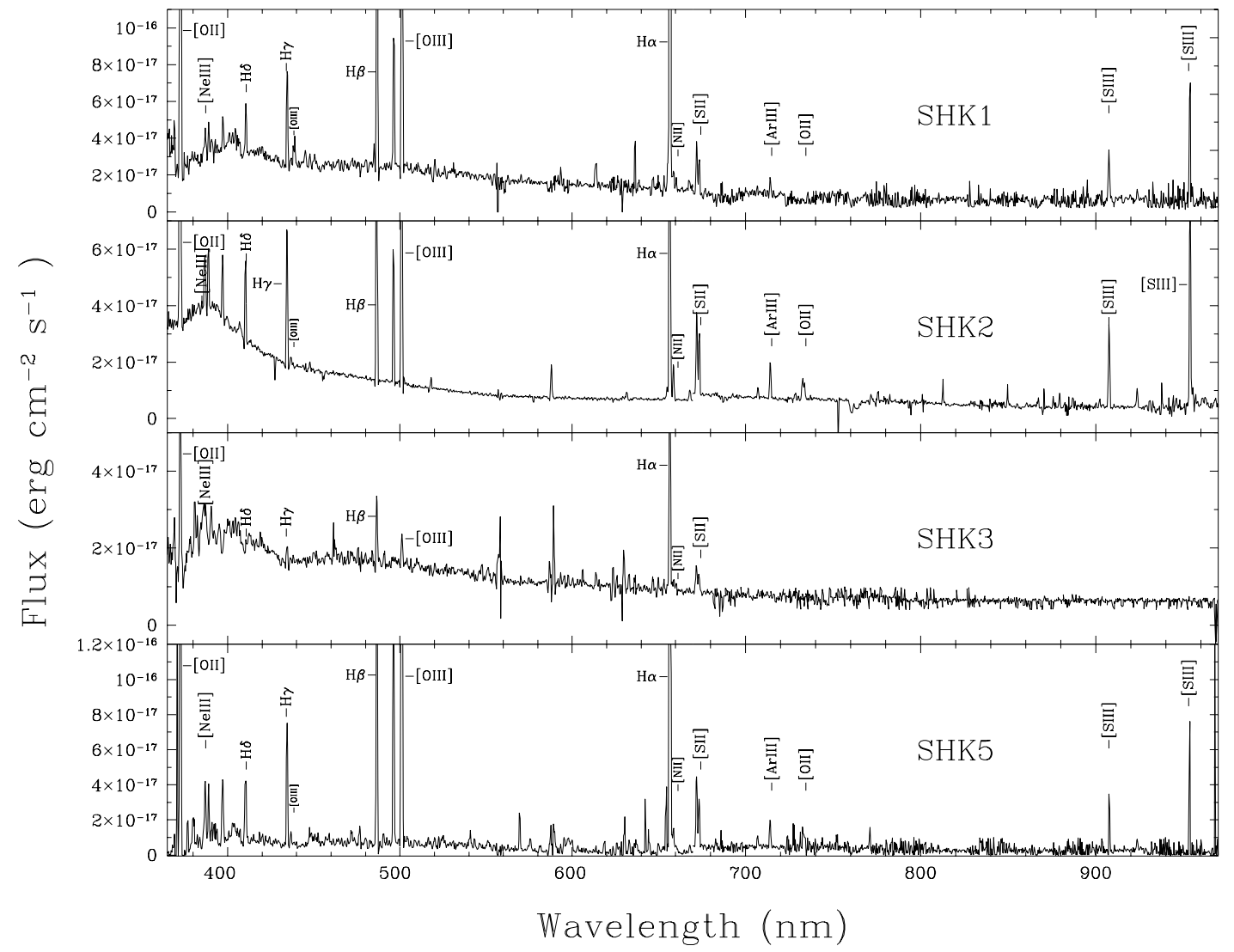

Fig. 6. VLT spectra of four H II regions in Sextans B.

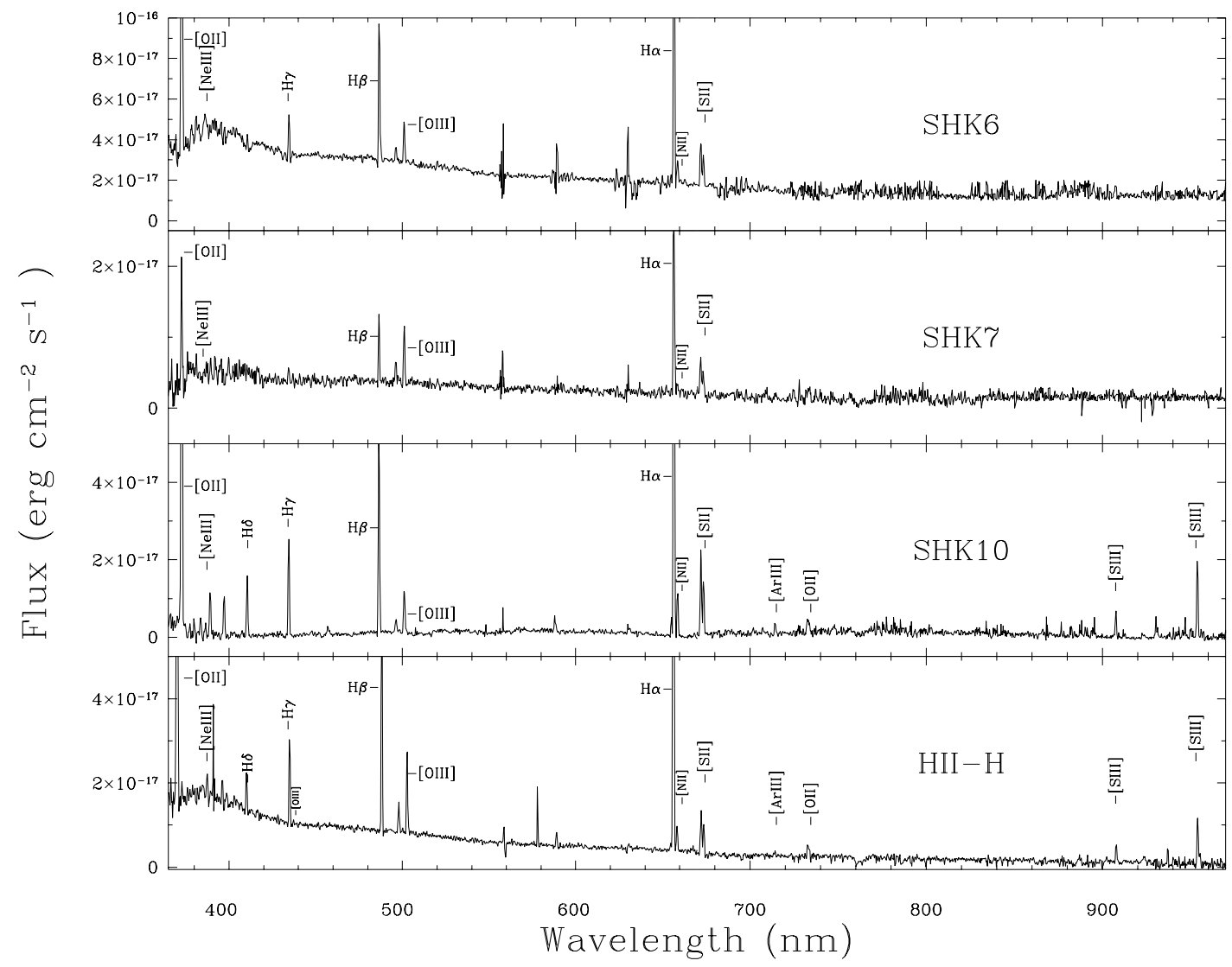

Fig. 7. VLT spectra of another four H II regions in Sextans B. 
slit centre, assuming a Gaussian point-spread functions with a FWHM which is the seeing of the observing night, and including seeing worsening toward bluer wavelengths.

As expected, the differential light-losses are negligible in the red side of the spectra, while become as large as $75 \%$ for Sextans A, and $90 \%$ for Sex B, at the shortest wavelength that we consider, which is that of the [O II] 372.7 doublet. At $\mathrm{H} \gamma$, they become as small as $20 \%$ and $25 \%$, respectively. We have applied these correction factors to the fluxes of blue lines, and attached an associated error of one third of the correction factor, which is propagated in all quantities (ionic abundances, ionization correction factor) derived using the blue lines. Note that the correction for the interstellar reddening applied to the spectra would by itself at least partially compensates for the atmospheric refraction, by "forcing" the Balmer lines to have the theoretical unreddened flux ratios (although with an estimated extinction value $c_{\beta}$ larger than the real one). Thus for Sextans A the difference between the observed fluxes and the refraction corrected fluxes, both after the reddening correction, amounts to $50 \%$ at the wavelength of the [O II] 3727 doublet and negligible at $\mathrm{H} \gamma$ for Sextans A, and of $60 \%$ and $10 \%$, respectively, for Sextans B.

In addition, it is important to note that these quite large corrections at the bluest wavelengths do not affect significantly our chemical analysis. In fact, the oxygen total and ionic abundances of the PNe where the [O II] 372.7 lines were measured change only by a factor much smaller than $0.1 \mathrm{dex}$, with respect to the values calculated using the observed fluxes. Moreover, once the fluxes were corrected for the effect of the atmospheric refraction, we analyzed the differences of $\mathrm{O}^{+} / \mathrm{O}$ derived from either the [O II] 372.7 or 733.0 lines, in those PNe which have both doublets measured. The differences resulted to be less than $15 \%$, which implies in the worst case, i.e. in the PN of Sextans A, a difference in the total oxygen abundance of only 0.05 dex.

For extended objects like H II regions, the effect of atmospheric dispersion is in principle more complex, because light is lost from one side of the slit but is "gained" from the other side. However, the extension of the spectra along the slit length does not show significant changes in surface brightness and excitation on scales of $\sim 1.5$ arcsec (after seeing convolution), which is the size of the displacement due to the atmospheric dispersion. Assuming that the HII regions are also similarly homogeneous in the direction perpendicular to the slit at these scales, then the effect of the atmospheric dispersion is negligible at all wavelengths, and therefore no corrections have been applied to the observed nebular spectra.

Line fluxes and their errors are listed in Tables 2 and 3. Errors include the contributions of background noise, sky subtraction, and flux calibration uncertainties. In the case of PNe, errors include the correction for the atmospheric dispersion were applied; they range from $40 \%$ at $370 \mathrm{~nm}$ to less than $1 \%$ at $500 \mathrm{~nm}$.

Finally, the observed line fluxes were corrected for the effect of interstellar extinction, adopting Mathis (1990) law with $R_{V}=3.1 . c_{\beta}$, which is the logarithmic difference between the observed and de-reddened $\mathrm{H} \beta$ fluxes, was determined comparing the observed Balmer $I(\mathrm{H} \alpha) / I(\mathrm{H} \beta)$ ratio with its theoretical value. For the spectra with the higher signal to noise ratio, we considered also the comparison between the observed and theoretical Balmer $I(\mathrm{H} \beta) / I(\mathrm{H} \gamma)$ and $I(\mathrm{H} \gamma) / I(\mathrm{H} \delta)$ ratios. In these cases $c_{\beta}$ is the weighted average of the three determinations.

\subsection{Radial velocities}

High-resolution spectroscopic data of three PNe of Sextans B were taken on April, 2002, with AF2/WYFFOS, a multi-object, wide field, fibre spectrograph working at the prime focus of the $4.2 \mathrm{~m}$ WHT (La Palma). We used the Echelle grating with a central wavelength of $493.0 \mathrm{~nm}$ and a reciprocal dispersion of $0.028 \mathrm{~nm} / \mathrm{pixel}$. The total exposure time was $5400 \mathrm{~s}$. The reduction was done with the IRAF DOFIBER package.

The high resolution WHT spectra allowed us to measure the Doppler shift of the [O III] doublet lines, and in one case also of $\mathrm{H} \beta$, in three PNe of Sextans B, namely PN 1, PN 4 and PN 5. The heliocentric radial velocities are listed in Table 2. They are consistent, within the errors, with the heliocentric radial velocity of Sextans B (300 $\mathrm{km} \mathrm{s}^{-1}$ from NED) and with the H I observations by Huchtmeier et al. (2003), confirming that the PNe belong indeed to the galaxy.

\section{Chemical abundances of planetary nebulae and $\mathrm{H}$ II regions}

\subsection{ICF method}

Chemical abundances were derived from the spectra by means of Ionization Correction Factors (ICF) method, following Kingsburgh \& Barlow (1994), with evaluation of the errors as in Corradi et al. (1997) and Perinotto \& Corradi (1998). As mentioned above, the [O III] 436.3 emission line was always measurable in all PNe with a sufficiently high signal to noise ratio, thus their [O III] electron temperature, $T_{\mathrm{e}[\mathrm{O} \text { III] }}$, could be determined. These are listed in Table 2; their high values are typical of low-metallicity PNe (cf. Stasinska 2002). The [O III] 436.3 line was also measured in four $\mathrm{H}$ II regions of Sextans A and in three H II regions of Sextans B, while its upper limit was estimated in four $\mathrm{H}$ II regions of Sextans A and one of Sextans B. Another temperature diagnostic line, [N II] $575.5 \mathrm{~nm}$ line, was observed in the PN of Sextans A, and a corresponding electron temperature $T_{\mathrm{e}[\mathrm{N} \mathrm{II]}}$ was determined. In this object, $T_{\mathrm{e}[\mathrm{O} \text { III] }}$ was used in computing chemical abundances with the ICF method for ions which are ionized twice or more times, and $T_{\mathrm{e}[\mathrm{N} \mathrm{II]}}$ for species only ionized once. The large difference between $T_{\mathrm{e}[\mathrm{O} \text { III] }}$ and $T_{\mathrm{e}[\mathrm{N} \mathrm{II}]}$ is typical of high excitation PNe and it was also found in several Galactic PNe (i.e. McKenna et al. 1996). Kingsburgh \& Barlow (1994) examined how the ratio between $T_{\mathrm{e}[\mathrm{O} \text { III] }}$ and $T_{\mathrm{e}[\mathrm{N} \mathrm{III}}$ behaves with nebular excitation, plotting this ratio versus the He II 4686 flux. They found a linear relation between these two quantities, as expressed in Eq. (3) of their paper. The PN of Sextans A, which is a very high excitation object, follows this relationship.

The [S III] near-infrared emission lines were detected in $4 \mathrm{PNe}$ and $12 \mathrm{H}$ II regions. These lines allow a better accuracy in the measurement of sulphur abundance, especially at low metallicities where [S II] lines are extremely faint. 
Table 2. Observed line fluxes $F_{\lambda}$ of PNe in Sextans A and Sextans B, on a scale where $F(\mathrm{H} \beta)=100.0$, and after correcting for atmospheric dispersion effects. ":" indicates uncertain values, and "<" upper limits. Associated errors are described in the text. Some nebular quantities are also reported: $c_{\beta}$, the electron density and temperatures $T_{\mathrm{e}}([\mathrm{O} \mathrm{III}])$ and $T_{\mathrm{e}}([\mathrm{N} \mathrm{II}])$, and the heliocentric radial velocity of three PNe where it was measured.

\begin{tabular}{|c|c|c|c|c|c|c|}
\hline Ion & SexA-PN & SexB-PN1 & SexB-PN2 & SexB-PN3 & SexB-PN4 & SexB-PN5 \\
\hline$c_{\beta}$ & $0.10 \pm 0.01$ & $0.10 \pm 0.02$ & $0.02 \pm 0.02$ & $0.13 \pm 0.01$ & $0.17 \pm 0.03$ & $0.22 \pm 0.01$ \\
\hline$N_{\mathrm{e}}\left(\mathrm{cm}^{-3}\right)$ & $2700 \pm 100$ & - & - & - & - & - \\
\hline$T_{\mathrm{e}}([\mathrm{O} \mathrm{III}])(\mathrm{K})$ & $22300 \pm 1000$ & $12800 \pm 2000$ & $16000 \pm 3000$ & $20000 \pm 2000$ & $11300 \pm 3000$ & $12800 \pm 1000$ \\
\hline$T_{\mathrm{e}}([\mathrm{N} \mathrm{II}])(\mathrm{K})$ & $13400 \pm 500$ & - & - & - & - & - \\
\hline$V\left(\mathrm{~km} \mathrm{~s}^{-1}\right)$ & - & $310 \pm 15$ & - & - & $310 \pm 15$ & $310 \pm 15$ \\
\hline $372.7[\mathrm{O} \mathrm{II}]$ & $176 . \pm 70$ & 18: & - & - & - & $21 . \pm 10$ \\
\hline $383.5 \mathrm{H} 9$ & $8 . \pm 4$ & - & $8 . \pm 6$ & $11 . \pm 6$ & - & - \\
\hline $386.8[\mathrm{Ne} \mathrm{III]}$ & $19 . \pm 6$ & $38 . \pm 10$ & $28 . \pm 7$ & $55 . \pm 5$ & $43 . \pm 7$ & $93 . \pm 5$ \\
\hline $388.9 \mathrm{He} \mathrm{I,} \mathrm{H8}$ & $28 . \pm 5$ & $16 . \pm 8$ & $21 . \pm 6$ & $32 . \pm 5$ & $24 . \pm 5$ & $16 . \pm 4$ \\
\hline 396.7 [Ne III], H7 & $27 . \pm 3$ & $29 . \pm 7$ & $22 . \pm 7$ & $29 . \pm 4$ & $37 . \pm 5$ & $46 . \pm 3$ \\
\hline $410.1 \mathrm{H} \delta$ & $31 . \pm 2$ & $25 . \pm 7$ & 19. \pm 6 & $26 . \pm 4$ & $26 . \pm 7$ & $23 . \pm 3$ \\
\hline $434.0 \mathrm{H} \gamma$ & $48 . \pm 2$ & $48 . \pm 4$ & $45 . \pm 4$ & $51 . \pm 3$ & $51 . \pm$ & $45 . \pm 1.6$ \\
\hline $436.3[\mathrm{O}$ III] & $23 . \pm 2$ & $8 . \pm 4$ & $13 . \pm 4$ & $16 . \pm 4$ & $7 . \pm 5$ & $11 . \pm 1.9$ \\
\hline $447.1 \mathrm{He} \mathrm{I}$ & $1.3 \pm 1$ & $5 . \pm 4$ & $9 . \pm 3$ & 4. \pm 2 & - & $5 . \pm 2$ \\
\hline 468.6 He II & $51 . \pm 2$ & $<1$ & $<3$ & $<2$ & $<1$ & $6 . \pm 2$ \\
\hline $471.2 \mathrm{He} \mathrm{I},[\mathrm{Ar} \mathrm{IV}]$ & - & - & - & $2 . \pm 1$ & - & - \\
\hline 474.0 [Ar IV] & - & - & - & $3 . \pm 2$ & - & - \\
\hline $486.1 \mathrm{H} \beta$ & $100 . \pm 1$ & $100 . \pm 4$ & $100 . \pm 4$ & $100 . \pm 1.5$ & $100 . \pm 5$ & $100 . \pm 2$ \\
\hline 495.9 [O III] & $206 . \pm 1$ & $202 . \pm 3$ & $190 . \pm 4$ & $182 . \pm 1.6$ & $256 . \pm 3$ & $316 . \pm 1.8$ \\
\hline 500.7 [O III] & $611 . \pm 1$ & $590 . \pm 2$ & $570 . \pm 3$ & $474 . \pm 1$ & $798 . \pm 3$ & $940 . \pm 1.5$ \\
\hline $520.0[\mathrm{~N} \mathrm{I}]$ & $38 . \pm 1$ & - & - & - & - & - \\
\hline $541.1 \mathrm{He}$ II & $4.2 \pm 1$ & - & - & - & - & - \\
\hline $575.5[\mathrm{~N} \mathrm{II}]$ & $24 . \pm 1$ & - & - & - & - & \\
\hline $587.6 \mathrm{He} \mathrm{I}$ & $8 . \pm 1$ & $16 . \pm 2$ & $17 . \pm 5$ & $15 . \pm 1$ & $15 . \pm 3$ & $22 . \pm 2$ \\
\hline 630.0 [O I] & $71 . \pm 1$ & $3 . \pm 2$ & $3 . \pm 1$ & - & - & - \\
\hline $636.3[\mathrm{O} \mathrm{I}]$ & $24 . \pm 1$ & - & - & - & - & - \\
\hline 654.8 [N II] & $291 . \pm 1$ & - & - & - & - & - \\
\hline $656.3 \mathrm{H} \alpha$ & $306 . \pm 1.5$ & $302 . \pm 2$ & $290 . \pm 3$ & $312 . \pm 1.3$ & $321 . \pm 3$ & $332 . \pm 1.3$ \\
\hline 658.4 [N II] & $835 . \pm 1.8$ & $2:$ & 7: & $6 . \pm 1.8$ & $4 . \pm 3$ & $12 . \pm 1.2$ \\
\hline $667.8 \mathrm{He} \mathrm{I}$ & $3 . \pm 1.6$ & $3 . \pm 2$ & $3 . \pm 1.5$ & 4. \pm 1 & - & $3 . \pm 1.5$ \\
\hline 671.7 [S II] & $5 . \pm 1.6$ & - & - & - & - & - \\
\hline 673.1 [S II] & $8 . \pm 1.5$ & - & - & - & - & - \\
\hline $706.5 \mathrm{He} \mathrm{I}$ & 4. \pm 1.6 & $6 . \pm 3$ & - & $10 . \pm 4$ & 4. \pm 2 & $3 . \pm 1.5$ \\
\hline 713.5 [Ar III] & $3 . \pm 3$ & - & - & $3 . \pm 2$ & - & - \\
\hline $728.1 \mathrm{He} \mathrm{I}$ & $2 . \pm 2$ & - & - & - & - & - \\
\hline 732.5 [O II] & $28 . \pm 2$ & $5 . \pm 3$ & 7. \pm 3 & - & $11 . \pm 2$ & - \\
\hline 906.9 [S III] & $7 . \pm 3$ & - & - & - & $2 . \pm 2$ & - \\
\hline 953.2 [S III] & $19 . \pm 2$ & - & - & 5: & 5: & 17: \\
\hline
\end{tabular}


Table 3. Observed line fluxes $F_{\lambda}$, on a scale where $F(\mathrm{H} \beta)=100.0$, and nebular parameters of the H II regions in Sextans A and Sextans B. Symbols are as in Table 1.

\begin{tabular}{|c|c|c|c|c|c|c|c|c|c|}
\hline SexA & HSK2 & HSK3 & HSK7 & HSK9 & HSK14 & HSK16 & HSK19 & HSK23 & HSK24 \\
\hline$c_{\beta}$ & $0.0 \pm 0.01$ & $0.23 \pm 0.01$ & $0.13 \pm 0.01$ & $0.0 \pm 0.01$ & $0.05 \pm 0.02$ & $0.14 \pm 0.01$ & $0.05 \pm 0.01$ & $0.21 \pm 0.01$ & $0.0 \pm 0.02$ \\
\hline$N_{\mathrm{e}}\left(\mathrm{cm}^{-3}\right)$ & $100 \pm 100$ & $200 \pm 100$ & $<100$ & $400 \pm 200$ & $<100$ & $<100$ & $<100$ & $<100$ & $100 \pm 100$ \\
\hline$T_{\mathrm{e}}([\mathrm{O}$ III $])(\mathrm{K})$ & $<12000$ & $13000 \pm 3000$ & $17000 \pm 4000$ & 14000: & $14000 \pm 1000$ & $<14000$ & $<13000$ & - & $<13000$ \\
\hline \multicolumn{10}{|l|}{ Ion } \\
\hline 372.7 [O II] & $240 . \pm 10$ & $126 . \pm 10$ & $205 . \pm 10$ & $145 . \pm 9$ & $145 . \pm 16$ & $213 . \pm 10$ & $179 . \pm 8$ & $194 . \pm 8$ & $160 . \pm 10$ \\
\hline 386.8 [Ne III] & $11 . \pm 5$ & $15 . \pm 8$ & $8 . \pm 6$ & $10 . \pm 6$ & $28 . \pm 8$ & $8 . \pm 5$ & $15 . \pm 5$ & $5 . \pm 3$ & $22 . \pm 8$ \\
\hline $388.9 \mathrm{He} \mathrm{I,} \mathrm{H8}$ & - & - & $9 . \pm 5$ & $15 . \pm 5$ & $21 . \pm 7$ & $15 . \pm 5$ & $8 . \pm 4$ & $10 . \pm 3$ & $18 . \pm 7$ \\
\hline 396.7 [Ne III], H7 & - & - & $14 . \pm 3$ & 10: & - & $20 . \pm 4$ & $17 . \pm 3$ & $9 . \pm 2$ & $20 . \pm 8$ \\
\hline $410.1 \mathrm{H} \delta$ & $24 . \pm 3$ & $20 . \pm 5$ & $25 . \pm 3$ & $22 . \pm 3$ & $26 . \pm 3$ & $21 . \pm 2$ & $25 . \pm 2$ & $25 . \pm 2$ & $28 . \pm 8$ \\
\hline $434.0 \mathrm{H} \gamma$ & $42 . \pm 2$ & $35 . \pm 2$ & $46 . \pm 3$ & $41 . \pm 3$ & $47 . \pm 3$ & 46. \pm 2 & $42 . \pm 2$ & $43 . \pm 2$ & $47 . \pm 5$ \\
\hline 436.3 [O III] & $<1$ & $2 \pm 2$ & $3 \pm 3$ & $2 . \pm 3$ & $6 . \pm 3$ & $<1$ & $<1$ & - & $<1$ \\
\hline $468.6 \mathrm{He}$ II & $<1$ & $<1$ & $<1$ & $<1$ & 3: & $6:$ & $<1$ & $<1$ & $<1$ \\
\hline $486.1 \mathrm{H} \beta$ & $100 . \pm 2$ & $100 . \pm 2$ & $100 . \pm 2$ & $100 . \pm 2$ & $100 . \pm 3$ & $100 . \pm 2$ & $100 . \pm 1$ & $100 . \pm 2$ & $100 . \pm 3$ \\
\hline 495.9 [O III] & $27 . \pm 2$ & $57 . \pm 2$ & $39 . \pm 2$ & $31 . \pm 2$ & $115 . \pm 3$ & $22 . \pm 2$ & $24 . \pm 1$ & $5 . \pm 3$ & $25 . \pm 3$ \\
\hline 500.7 [O III] & $84 . \pm 2$ & $159 . \pm 2$ & $120 . \pm 2$ & $94 . \pm 2$ & $340 . \pm 3$ & $65 . \pm 2$ & $72 . \pm 1.5$ & $15 . \pm 2$ & $75 . \pm 3$ \\
\hline $587.6 \mathrm{He} \mathrm{I}$ & - & 7. \pm 2 & $10 . \pm 2$ & $4 . \pm 2$ & - & - & - & - & - \\
\hline 630.0 [O I] & - & - & - & - & $8 . \pm 2$ & - & - & - & - \\
\hline $656.3 \mathrm{H} \alpha$ & $281 . \pm 2$ & $335 . \pm 2$ & $312 . \pm 2$ & $281 . \pm 2$ & $295 . \pm 3$ & $314 . \pm 2$ & $295 . \pm 3$ & $329 . \pm 2$ & $281 . \pm 3$ \\
\hline 658.4 [N II] & $7 . \pm 2$ & $9 . \pm 3$ & $9 . \pm 2$ & $11 . \pm 2$ & $7 . \pm 3$ & $8 . \pm 2$ & $10 . \pm 3$ & $13 . \pm 2$ & $10 . \pm 3$ \\
\hline 671.7 [S II] & $18 . \pm 2$ & $16 . \pm 3$ & $17 . \pm 2$ & $17 . \pm 2$ & $17 . \pm 3$ & $24 . \pm 2$ & $26 . \pm 2$ & $22 . \pm 2$ & $26 . \pm 3$ \\
\hline 673.1 [S II] & $14 . \pm 2$ & $13 . \pm 3$ & $11 . \pm 2$ & $15 . \pm 2$ & $11 . \pm 3$ & $16 . \pm 2$ & $16 . \pm 2$ & $14 . \pm 2$ & $20 . \pm 3$ \\
\hline 713.5 [Ar III] & 4. \pm 2 & $5 . \pm 3$ & $5 . \pm 2$ & $3 . \pm 2$ & $3 . \pm 3$ & $6 . \pm 2$ & $2 . \pm 2$ & $2 . \pm 2$ & $1 . \pm 3$ \\
\hline 732.5 [O II] & - & $13 . \pm 3$ & $8 . \pm 2$ & - & $14 . \pm 3$ & - & - & $8 . \pm 2$ & - \\
\hline 906.9 [S III] & $5 . \pm 2$ & $18 . \pm 3$ & $16 . \pm 3$ & $7 . \pm 5$ & $8 . \pm 2$ & $20 . \pm 3$ & - & $8 . \pm 2$ & - \\
\hline 953.2 [S III] & $14 . \pm 2$ & $50 . \pm 3$ & $42 . \pm 5$ & $18 . \pm 5$ & - & - & - & - & - \\
\hline SexB & SHK1 & SHK2 & SHK3 & SHK5 & SHK6 & SHK7 & SHK10 & Н II H & \\
\hline$c_{\beta}$ & $0.0 \pm 0.02$ & $0.13 \pm 0.01$ & $0.30 \pm 0.05$ & $0.16 \pm 0.01$ & $0.30 \pm 0.03$ & $0.12 \pm 0.02$ & $0.29 \pm 0.01$ & $0.15 \pm 0.01$ & \\
\hline$N_{\mathrm{e}}\left(\mathrm{cm}^{-3}\right)$ & $<100$ & $200 \pm 100$ & $<100$ & $<100$ & $<100$ & $300 \pm 100$ & $<100$ & $<100$ & \\
\hline$T_{\mathrm{e}}([\mathrm{O} \mathrm{III}])(\mathrm{K})$ & $13000:$ & $15000:$ & - & $12000 \pm 2000$ & - & - & - & $<17000$ & \\
\hline \multicolumn{10}{|l|}{ Ion } \\
\hline 372.7 [O II] & $181 . \pm 7$ & $350 . \pm 4$ & $390 . \pm 30$ & $182 . \pm 8$ & $210 . \pm 40$ & $324 . \pm 10$ & $413 . \pm 6$ & $292 . \pm 7$ & \\
\hline 386.8 [Ne III] & $9 . \pm 3$ & $29 . \pm 3$ & - & $17 . \pm 4$ & - & - & $7 . \pm 3$ & $9 . \pm 3$ & \\
\hline 388.9 He I, H8 & $8 . \pm 2$ & $26 . \pm 2$ & - & $14 . \pm 3$ & - & - & $23 . \pm 2$ & $11 . \pm 3$ & \\
\hline 396.7 [Ne III], H7 & $10 . \pm 2$ & $27 . \pm 1$ & - & $20 . \pm 2$ & - & - & $20 . \pm 2$ & $12 . \pm 3$ & \\
\hline $410.1 \mathrm{H} \delta$ & $13 . \pm 2$ & $34 . \pm 1$ & - & $22 . \pm 2$ & - & - & $30 . \pm 2$ & $19 . \pm 2$ & \\
\hline $434.0 \mathrm{H} \gamma$ & $27 . \pm 2$ & $53 . \pm 1$ & - & $38 . \pm 2$ & - & - & $47 . \pm 2$ & $40 . \pm 2$ & \\
\hline 436.3 [O III] & $2 . \pm 2$ & $3 . \pm 2$ & - & $3 . \pm 2$ & - & - & - & $<1$ & \\
\hline 468.6 He II & $<1$ & $<1$ & - & $<1$ & - & $<1$ & $<1$ & - & \\
\hline $486.1 \mathrm{H} \beta$ & $100 . \pm 3$ & $100 . \pm 0.5$ & $100 . \pm 9$ & $100 . \pm 2$ & $100 . \pm 5$ & $100 . \pm 4$ & $100 . \pm 1$ & $100 . \pm 1$ & \\
\hline 495.9 [O III] & $50 . \pm 3$ & $55 . \pm 0.5$ & $17 . \pm 7$ & $86 . \pm 2$ & $35 . \pm 5$ & $12 . \pm 4$ & $9 . \pm 1$ & $15 . \pm 1$ & \\
\hline 500.7 [O III] & $147 . \pm 3$ & $148 . \pm 0.5$ & $52 . \pm 7$ & $264 . \pm 2$ & $113 . \pm 5$ & $30 . \pm 4$ & $23 . \pm 1$ & 38. \pm 1 & \\
\hline $587.6 \mathrm{He} \mathrm{I}$ & - & $16 . \pm 0.5$ & - & $5 . \pm$ & - & - & - & $7 . \pm 1$ & \\
\hline $656.3 \mathrm{H} \alpha$ & $272 . \pm 2$ & $312 . \pm 0.5$ & $351 . \pm 4$ & $319 . \pm 1.5$ & $351 . \pm 8$ & $310 . \pm 2$ & $350 . \pm 0.5$ & $316 . \pm 1$ & \\
\hline 658.4 [N II] & $5 . \pm 2$ & $15 . \pm 0.5$ & $25 . \pm 4$ & $8 . \pm 1$ & $28 . \pm 7$ & $23 . \pm 2$ & $22 . \pm 1$ & $10 . \pm 1$ & \\
\hline $667.8 \mathrm{He}$ I & - & $5 . . \pm 0.5$ & - & - & - & - & - & - & \\
\hline 671.7 [S II] & $15 . \pm 2$ & $27 . \pm 1$ & $59 . \pm 4$ & $21 . \pm 2$ & $75 . \pm 8$ & $29 . \pm 3$ & $33 . \pm 1$ & $22 . \pm 1$ & \\
\hline $673.1[\mathrm{~S} \mathrm{II}]$ & $9 . \pm 2$ & $22 . \pm 1$ & $43 . \pm 4$ & $15 . \pm 2$ & $53 . \pm 8$ & $24 . \pm 3$ & $21 . \pm 1$ & $16 . \pm 1$ & \\
\hline $706.5 \mathrm{He} \mathrm{I}$ & - & $4 . \pm 1$ & - & - & - & - & - & - & \\
\hline 713.5 [Ar III] & $4 . \pm 2$ & $12 . \pm 1$ & - & $2 . \pm 2$ & - & $6 . \pm 3$ & $5 . \pm 1$ & $2 . \pm 1$ & \\
\hline 732.5 [O II] & - & $13 . \pm 1$ & - & $9 . \pm 2$ & - & - & $12 . \pm 2$ & $9 . \pm 1$ & \\
\hline 906.9 [S III] & $10 . \pm 3$ & $16 . \pm 1$ & - & $18 . \pm 2$ & - & - & $12 . \pm 2$ & $8 . \pm 2$ & \\
\hline 953.2 [S III] & $24 . \pm 3$ & $41 . \pm 1$ & - & $43 . \pm 2$ & - & - & $36 . \pm 2$ & $21 . \pm 2$ & \\
\hline
\end{tabular}


The ICF method was applied to the whole sample of PNe and to $\mathrm{H}$ II regions where $T_{\mathrm{e}[\mathrm{O} \text { III] }}$ was measured. Formal errors on the ICF abundances were computed taking into account the uncertainties in the observed fluxes and in the electron temperatures. A discussion of the error propagation computed with the ICF method is in Corradi et al. (1997).

\subsection{Modelling with CLOUDY}

$\mathrm{PNe}$ and $\mathrm{H}$ II regions were also modelled using the photoionization code CLOUDY 94.00 (Ferland et al. 1998), assuming a blackbody central star with effective temperature derived using the Ambartsumian's (1932) or Gurzadyan's (1988) methods, and a spherical nebula with constant density. Density was derived from the [S II] 671.7/673.1 flux ratio, which was measured in all $\mathrm{H}$ II regions and in the PN of Sextans A. For $\mathrm{PNe}$ in Sextans B, it was assumed equal to $3000 \mathrm{~cm}^{-3}$, which can be considered a typical value for Galactic PNe (see e.g. Stanghellini \& Kaler 1989). In addition, we made some tests varying the density of $\pm 1000 \mathrm{~cm}^{-3}$ with both the ICF and CLOUDY methods, obtaining negligible changes in the computed abundances.

In the model, we include dust typical of Galactic PNe and of the Orion nebula, for PNe and $\mathrm{H}$ II regions, respectively. The accuracy of the CLOUDY method was tested in Magrini et al. (2004), who applied it to seven bright Galactic PNe using only the emission lines typically present in extragalactic PN spectra, and compared the results with the chemical abundances obtained with the ICF method by KB94. A good agreement was found in the helium and metals abundances. For further details on the modelling procedure see Magrini et al. (2004) and Perinotto et al. (2004a).

\subsection{Results}

The chemical abundances derived for the current sample with the ICF and CLOUDY methods are in general agreement with each other, showing the following rms differences in both galaxies: \pm 0.01 for $\mathrm{He} / \mathrm{H}$ (linear), \pm 0.15 dex for $\mathrm{N} / \mathrm{H}, \pm 0.1 \mathrm{dex}$ for $\mathrm{O} / \mathrm{H}, \pm 0.1 \mathrm{dex}$ for $\mathrm{Ne} / \mathrm{H}, \pm 0.1 \mathrm{dex}$ for $\mathrm{S} / \mathrm{H}, \pm 0.1 \mathrm{dex}$ for $\mathrm{Ar} / \mathrm{H}$ for both $\mathrm{PNe}$ and $\mathrm{H}$ II regions.

The total and ionic abundances, and the ICF of $\mathrm{PNe}$ and $\mathrm{H}$ II regions are reported in Tables 4 and 5. The total abundances for the whole sample of objects computed with both methods, and their mean values are shown in Table 6 for PNe and in Tables 8 and 9 for $\mathrm{H}$ II regions. H II regions where only the CLOUDY method was applied because of the absence of a reliable determination of the electron temperature are marked with a "*" in Tables 8 and 9. For Sextans B PN 3, ICF(N) and $\operatorname{ICF}(\mathrm{S})$ were not computed because the [O II] lines needed to compute them were not detected, and thus for these elements only the results from CLOUDY are quoted.

In the case of $\mathrm{H}$ II regions, the ICF of He does not take into account the neutral helium, which might be a considerable fraction of the total helium abundance. However, for the $\mathrm{H}$ II regions where $\left.T_{\mathrm{e}[\mathrm{O}} \mathrm{III}\right]$ was measured, and therefore where the abundances with the ICF method were computed (HKS3,
HKS7, HKS9, HKS14 of Sextans A and SHK1, SHK2, SHK5 of Sextans B), the comparison between the results of ICF and CLOUDY methods shows that the mean contribution of neutral helium is smaller than $20 \%$. This relatively small contribution makes unnoticeable the difference between the two results within our present errors.

According to the definition by Kingsburgh \& Barlow (1994) for Galactic PNe, the PN in Sextans A would be classified as a type I PNe in the Peimbert scheme $(\log (\mathrm{N} / \mathrm{O})>-0.1)$, while all PNe in Sextans B would be non-Type I. However, it is clear that this distinction is hardly applicable to extragalactic $\mathrm{PNe}$, which span a much larger range of metallicities.

The modelling with CLOUDY also allowed us to estimate some stellar parameters as the luminosity and temperature of the exciting stars, as described by Magrini et al. (2004). We remind briefly how $T_{\star}$ and $L_{\star}$ were computed in the modelling with CLOUDY. The luminosity of the central star was set to reproduce the observed absolute flux of the [O III] $\lambda 5007$ nebular line, measured by Magrini et al. $(2002,2003)$ via aperture photometry, and reddening-corrected, while the BB $T_{\star}$ was set to match the $\mathrm{He} \mathrm{I} / \mathrm{He}$ II line ratio.

Using these parameters, the PNe were located in the $\log T_{\star}-\log L_{\star}$ diagram and compared with the available H-burning and He-burning evolutionary tracks for $Z=0.004$ from Vassiliadis \& Wood (1994), as shown in Fig. 8. The obtained $T_{\star}$ and $\log \left(L_{\star} / L_{\odot}\right)$ are reported in Table 7 . Typical errors obtained with the CLOUDY modelling are of the order of 0.15 for $\log T_{\star}$ and of 0.25 for $\log L_{\star}$.

The mass of the core of the PN in Sextans A is distinctively larger than for the PNe in Sextans B, and would imply a progenitor initial mass of $\sim 2-3.5 M_{\odot}$, using the initial-to-final mass relation adopted by Vassiliadis \& Wood (1994). The relatively large mass of the progenitor is also typical of type I Galactic PNe (Corradi \& Schwarz 1995).

The PNe of Sextans B can be instead associated with less massive cores. Among them, PN 5 seems to have the most massive one. Note, however, that the metallicity of Sextans A and Sextans B, 0.001 and 0.002 , respectively, is significantly lower than for the models of Vassiliadis \& Wood (1994), and therefore evolutionary tracks for such low metallicities would be needed for a deeper analysis. From Fig. 8 and the initial to final mass relationship used by Vassiliadis \& Wood (1994), we find that the central stars of Sex B PNe had initial masses between 0.9 and $1.5 M_{\odot}$. Stars with these masses and with an initial metallicity $Z=0.001$ were born between about 10 and 2 Gyr ago (cf. Charbonnel et al. 1999, 1996). As the mean oxygen abundance of PNe in Sextans B is equal, within the errors, to that of $\mathrm{H}$ II regions, this would mean that no noticeable chemical enrichment has occurred in the galaxy since long time ago.

\subsection{Comparison with previous determinations of chemical abundances}

In Sextans A, Skillman et al. (1989) observed spectroscopically four $\mathrm{H}$ II regions, deriving $12+\log \mathrm{O} / \mathrm{H}=7.49$. The same spectroscopic data were used by Richer \& McCall (1995) 
Table 4. Total and ionic chemical abundances, and ionization correction factors of Sextans A and Sextans B PNe, computed with the ICF method.

\begin{tabular}{|c|c|c|c|c|c|c|}
\hline & SexA-PN & SexB-PN1 & SexB-PN2 & SexB-PN3 & SexB-PN4 & SexB-PN5 \\
\hline $\mathrm{He}^{+} / \mathrm{H}^{+} \times 10^{2}$ & $3.6 \pm 1.1$ & $9.8 \pm 1.5$ & $9.3 \pm 1.5$ & $7.4 \pm 1.5$ & $9.4 \pm 1.3$ & $12.8 \pm 2$ \\
\hline $\mathrm{He}^{++} / \mathrm{H}^{+} \times 10^{2}$ & $4.8 \pm 0.5$ & $0.09 \pm 0.05$ & $0.3 \pm 0.1$ & $0.2 \pm 0.1$ & $0.1 \pm 0.1$ & $0.5 \pm 0.1$ \\
\hline $\mathrm{He} / \mathrm{H} \times 10^{2}$ & $8.4 \pm 1.6$ & $9.9 \pm 1.5$ & $9.6 \pm 1.6$ & $7.6 \pm 1.6$ & $0.95 \pm 1.4$ & $13.3 \pm 2.1$ \\
\hline $\mathrm{N}^{0} / \mathrm{H}^{+} \times 10^{5}$ & $3.9 \pm 1.2$ & - & - & - & - & - \\
\hline $\mathrm{N}^{+} / \mathrm{H}^{+} \times 10^{5}$ & $7.1 \pm 0.9$ & $0.008:$ & 0.003: & $0.03 \pm 0.01$ & $0.015 \pm 0.01$ & $0.04 \pm 0.02$ \\
\hline $\operatorname{ICF}(\mathrm{N})$ & 3.0 & 72. & 19. & - & 45. & 130. \\
\hline $\mathrm{N} / \mathrm{H} \times 10^{5}$ & $22 . \pm 10$ & 0.5 : & 0.5 : & $>0.03$ & $0.7 \pm 0.5$ & $5 . \pm 3$ \\
\hline $\mathrm{O}^{0} / \mathrm{H}^{+} \times 10^{5}$ & $5.2 \pm 1.1$ & $0.06 \pm 0.01$ & $0.07 \pm 0.01$ & - & - & - \\
\hline $\mathrm{O}^{+} / \mathrm{H}^{+} \times 10^{5}$ & $4.3 \pm 1.6$ & $0.13 \pm 0.30$ & $0.27 \pm 0.30$ & - & $0.4 \pm 0.9$ & $0.1 \pm 0.2$ \\
\hline $\mathrm{O}^{++} / \mathrm{H}^{+} \times 10^{5}$ & $3.2 \pm 0.3$ & $9.4 \pm 3.6$ & $4.8 \pm 3.5$ & $3.4 \pm 1.9$ & $17.3 \pm 4$ & $15.9 \pm 4$ \\
\hline $\mathrm{ICF}(\mathrm{O})$ & 1.7 & 1.0 & 1.0 & 1.0 & 1.0 & 1.0 \\
\hline $\mathrm{O} / \mathrm{H} \times 10^{5}$ & $13.0 \pm 3.7$ & $9.6 \pm 3.7$ & $5.2 \pm 3.9$ & $3.5 \pm 2.4$ & $17.8 \pm 5$ & $16.4 \pm 5$ \\
\hline $\mathrm{Ne}^{++} / \mathrm{H}^{+} \times 10^{6}$ & $9.2 \pm 3.1$ & $150 . \pm 80$ & $55 . \pm 30$ & $80 . \pm 60$ & $30 . \pm 10$ & $45 . \pm 20$ \\
\hline $\mathrm{ICF}(\mathrm{Ne})$ & 4.1 & 1.0 & 1.1 & 1.0 & 1.0 & 1.0 \\
\hline $\mathrm{Ne} / \mathrm{H} \times 10^{5}$ & $0.38 \pm 0.20$ & $1.6 \pm 1.2$ & $0.6 \pm 0.3$ & $0.8 \pm 0.6$ & $3 . \pm 1$ & $5 . \pm 3$ \\
\hline $\mathrm{S}^{+} / \mathrm{H}^{+} \times 10^{7}$ & $2.3 \pm 0.8$ & - & - & - & - & - \\
\hline $\mathrm{S}^{++} / \mathrm{H}^{+} \times 10^{7}$ & $3.9 \pm 0.8$ & - & - & $0.8 \pm 0.8$ & $0.9 \pm 0.8$ & $2.5 \pm 1$ \\
\hline $\operatorname{ICF}(S)$ & 1.1 & - & - & - & 2.5 & 3.5 \\
\hline $\mathrm{S} / \mathrm{H} \times 10^{7}$ & $7.0 \pm 1.9$ & - & - & $>0.8$ & $2 . \pm 1$ & $9 . \pm 3$ \\
\hline $\mathrm{Ar}^{++} / \mathrm{H}^{+} \times 10^{8}$ & $9.1 \pm 8.7$ & - & - & $19 . \pm 10$ & - & - \\
\hline $\mathrm{Ar}^{+++} / \mathrm{H}^{+} \times 10^{8}$ & - & - & - & $16 . \pm 10$ & - & - \\
\hline $\mathrm{ICF}(\mathrm{Ar})$ & 1.9 & - & - & 1.3 & - & - \\
\hline $\mathrm{Ar} / \mathrm{H} \times 10^{7}$ & $1.7 \pm 1.7$ & - & - & $5 . \pm 4$ & - & - \\
\hline
\end{tabular}

to recalculate $12+\log \mathrm{O} / \mathrm{H}=7.55 \pm 0.11$ and by Pilyugin (2001) who obtained through the P-method (Pilyugin 2000) $12+\log \mathrm{O} / \mathrm{H}=7.7$. An analysis of supergiant stars by Kaufer et al. (2004) derived a total metallicity which is 1.09 dex lower than solar. A red giants metallicity $[\mathrm{Fe} / \mathrm{H}]=-1.9 \pm 0.4$ was derived by Grebel (2000). Dolphin et al. (2003) found through modeling of the color-magnitude diagram a mean metallicity of $[\mathrm{M} / \mathrm{H}] \sim-1.4$. These values are in good agreement with $12+\log \mathrm{O} / \mathrm{H}=7.6 \pm 0.2$, the average oxygen abundance derived from our observations of nine $\mathrm{H}$ II regions, corresponding approximately to $[\mathrm{Fe} / \mathrm{H}]=-1.6$. The transformation from $[\mathrm{O} / \mathrm{H}]$ to $[\mathrm{Fe} / \mathrm{H}]$ needed to compare our nebular oxygen abundances with the stellar abundances was computed according to the empirical transformation by Mateo (1998). We used the solar abundances from Grevesse \& Sauval (1998) to derive $[\mathrm{O} / \mathrm{H}]$ from the measured $12+\log \mathrm{O} / \mathrm{H}$. The oxygen abundance of the PN in Sextans A is significantly higher $(12+\log \mathrm{O} / \mathrm{H}=8.0 \pm 0.1)$, and will be discussed in Sect. 4 .
In Sextans B, the observations by Skillman et al. (1989) of four $\mathrm{H}$ II regions gave $12+\log \mathrm{O} / \mathrm{H}=7.56$, while Moles et al. (1990) measured $12+\log \mathrm{O} / \mathrm{H}=8.1$ in a single $\mathrm{H}$ II region. Pilyugin (2001) recalculated both abundances obtaining $12+\log \mathrm{O} / \mathrm{H}=7.85$ and 7.87 , respectively. The average oxygen abundance derived in this paper with eight $\mathrm{H}$ II regions is $7.8 \pm 0.2$ and from five PNe is $8.0 \pm 0.3$, thus in agreement with Moles et al. (1990) and Pilyugin (2001).

A recent very work about spectroscopy of $\mathrm{H}$ II regions and PNe of Sextans A and Sextans B is by Kniazev et al. (2005). The authors derived chemical abundances of the PN and of three H II regions in Sextans A, and of one PN (PN3) and three H II regions in Sextans B with the ICF method. In Sextans A, they found an oxygen abundance of $8.02 \pm 0.05$ in the $\mathrm{PN}$ and $7.54 \pm 0.06$ in three $\mathrm{H}$ II regions, that, we did not observe. These H II regions belong to the same complex of HSK14, HSK16, and HSK19, for which we derived a mean $12+\log \mathrm{O} / \mathrm{H}=7.65 \pm 0.1$. In PN3 of Sextans B they 
Table 5. Total and ionic chemical abundances, and ionization correction factors of Sextans A and Sextans B H II regions, computed with the ICF method.

\begin{tabular}{|c|c|c|c|c|c|c|c|}
\hline & HKS3 & HKS7 & HKS9 & HKS14 & SHK1 & SHK2 & SHK5 \\
\hline $\mathrm{He}^{+} / \mathrm{H}^{+} \times 10^{2}$ & $5.0 \pm 1.0$ & $7.8 \pm 1.2$ & $3.0 \pm 0.5$ & - & - & $12.3 \pm 2$ & $3.5 \pm 1.0$ \\
\hline $\mathrm{He}^{++} / \mathrm{H}^{+} \times 10^{2}$ & $0.09 \pm 0.01$ & $0.09 \pm 0.01$ & $0.09 \pm 0.01$ & $0.3 \pm 0.1$ & $0.09 \pm 0.01$ & $0.08 \pm 0.01$ & $0.5 \pm 0.2$ \\
\hline $\mathrm{He} / \mathrm{H} \times 10^{2}$ & $5.1 \pm 1.0$ & $7.9 \pm 1.2$ & $3.1 \pm 0.5$ & - & - & $12.4 \pm 2$ & $4.0 \pm 1.2$ \\
\hline $\mathrm{N}^{+} / \mathrm{H}^{+} \times 10^{7}$ & $2.9 \pm 0.4$ & $3.3 \pm 0.5$ & $4.4 \pm 0.7$ & $2.7 \pm 0.4$ & $2.0 \pm 0.3$ & $6.0 \pm 0.1$ & $2.7 \pm 0.4$ \\
\hline $\operatorname{ICF}(\mathrm{N})$ & 5.5 & 2.4 & 2.6 & 7.2 & 4.7 & 2.8 & 8.7 \\
\hline $\mathrm{N} / \mathrm{H} \times 10^{7}$ & $15.7 \pm 3.0$ & $7.9 \pm 2.0$ & $12.0 \pm 4.0$ & $20 . \pm 7.0$ & $9.5 \pm 3.5$ & $17 . \pm 5$ & $24 . \pm 8$ \\
\hline $\mathrm{O}^{0} / \mathrm{H}^{+} \times 10^{5}$ & - & - & - & $0.2 \pm 0.1$ & - & - & - \\
\hline $\mathrm{O}^{+} / \mathrm{H}^{+} \times 10^{5}$ & $0.6 \pm 0.1$ & $0.7 \pm 0.1$ & $0.5 \pm 0.1$ & $0.6 \pm 0.1$ & $0.6 \pm 0.1$ & $1.0 \pm 0.1$ & $0.7 \pm 0.1$ \\
\hline $\mathrm{O}^{++} / \mathrm{H}^{+} \times 10^{5}$ & $2.7 \pm 0.9$ & $0.8 \pm 0.3$ & $0.9 \pm 0.3$ & $3.8 \pm 1.3$ & $2.3 \pm 0.8$ & $1.8 \pm 0.6$ & $4.3 \pm 1.5$ \\
\hline $\operatorname{ICF}(\mathrm{O})$ & 1.0 & 1.0 & 1.0 & 1.0 & 1.0 & 1.0 & 1.0 \\
\hline $\mathrm{O} / \mathrm{H} \times 10^{5}$ & $3.3 \pm 1.0$ & $1.5 \pm 0.4$ & $1.4 \pm 0.4$ & $4.5 \pm 1.5$ & $3.0 \pm 0.9$ & $2.8 \pm 0.7$ & $5.0 \pm 1.6$ \\
\hline $\mathrm{Ne}^{++} / \mathrm{H}^{+} \times 10^{6}$ & $7.0 \pm 2.0$ & $1.4 \pm 0.5$ & $2.1 \pm 0.8$ & $7.7 \pm 2.7$ & $3.5 \pm 1.3$ & $7.5 \pm 2.7$ & $8.1 \pm 2.9$ \\
\hline $\mathrm{ICF}(\mathrm{Ne})$ & 1.2 & 1.7 & 1.7 & 1.2 & 1.3 & 1.6 & 1.2 \\
\hline $\mathrm{Ne} / \mathrm{H} \times 10^{5}$ & $9.0 \pm 5.0$ & $2.4 \pm 1.3$ & $3.5 \pm 1.9$ & $8.9 \pm 5.0$ & $4.4 \pm 2.6$ & $1.8 \pm 0.6$ & $9.5 \pm 5.0$ \\
\hline $\mathrm{S}^{+} / \mathrm{H}^{+} \times 10^{7}$ & $1.4 \pm 0.2$ & $1.5 \pm 0.2$ & $2.0 \pm 0.3$ & $1.6 \pm 0.2$ & $1.3 \pm 0.1$ & $3.0 \pm 0.5$ & $1.80 \pm 0.2$ \\
\hline $\mathrm{S}^{++} / \mathrm{H}^{+} \times 10^{7}$ & $6.0 \pm 1.0$ & $6.0 \pm 1.7$ & $3.2 \pm 0.9$ & $3.5 \pm 0.9$ & $4.9 \pm 1.2$ & $7.5 \pm 2$ & $6.50 \pm 1.6$ \\
\hline $\operatorname{ICF}(S)$ & 1.3 & 1.1 & 1.1 & 1.4 & 1.25 & 1.1 & 1.5 \\
\hline $\mathrm{S} / \mathrm{H} \times 10^{7}$ & $9.2 \pm 2.0$ & $8.0 \pm 2.0$ & $5.7 \pm 1.3$ & $7.2 \pm 1.6$ & $7.8 \pm 1.9$ & $11.6 \pm 3$ & $12.2 \pm 3$ \\
\hline $\mathrm{Ar}^{++} / \mathrm{H}^{+} \times 10^{7}$ & $1.0 \pm 0.3$ & $0.7 \pm 0.2$ & $0.8 \pm 0.2$ & $0.8 \pm 0.2$ & $1.1 \pm 0.3$ & $3.2 \pm 0.4$ & $0.5 \pm 0.1$ \\
\hline $\mathrm{ICF}(\mathrm{Ar})$ & 1.9 & 1.9 & 1.9 & 1.9 & 1.9 & 1.9 & 1.9 \\
\hline $\mathrm{Ar} / \mathrm{H} \times 10^{7}$ & $1.9 \pm 0.7$ & $1.2 \pm 0.5$ & $1.5 \pm 0.5$ & $1.5 \pm 0.5$ & $2.1 \pm 0.7$ & $5.9 \pm 2$ & $0.9 \pm 0.3$ \\
\hline
\end{tabular}

derived $12+\log \mathrm{O} / \mathrm{H}=7.47 \pm 0.16$, in agreement with our value $7.5 \pm 0.2$. They also observed the same $\mathrm{H}$ II regions for which we derived chemical abundances with the ICF method, SHK1, SHK2, SHK5, finding $12+\log \mathrm{O} / \mathrm{H} 7.50 \pm 0.08,7.55 \pm 0.06$, $7.84 \pm 0.05$, respectively. These values are similar to our ICF determinations: $7.5 \pm 0.1,7.5 \pm 0.1,7.7 \pm 0.1$.

\subsection{Relationships between chemical abundances}

The relation between $\mathrm{He} / \mathrm{H}$ and N/O is shown in Fig. 9, where the observed chemical abundances of $\mathrm{PNe}$ are compared with new models by Marigo (2004, private communication) built for initial metallicities $Z=0.001$ and $Z=0.004$. Both models predict, for a progenitor mass below $3 M_{\odot}$, an enhancement of $\mathrm{O} / \mathrm{H}$ compared to $\mathrm{N} / \mathrm{H}$, producing a $\mathrm{N} / \mathrm{O}$ abundance ratio decreasing with $\mathrm{He} / \mathrm{H}$. At even higher mass progenitors, N/O increases quickly and significantly as the result of hot-bottom burning. The abundance of the PN of Sextans A is in good agreement with the model $Z=0.001$ and an initial mass around $4 M_{\odot}$. Four of Sextans B PNe match within $1-1.5 \sigma$ with the same model, $Z=0.001$, and masses between 0.55 and $3 M_{\odot}$; one of them, PN 5, is in better agreement with the model with $Z=0.004$ and a progenitor with initial mass of about $1.5 M_{\odot}$, in agreement with the result obtained with the evolutionary tracks (Fig. 8). Note however that the mean metallicity of Sextans B is $Z=0.002$.

Figure 10 shows the relationship between N/O and N/H for $\mathrm{PNe}$ in several nearby galaxies. This relation is well defined for $\mathrm{PNe}$ with a slope near to unity, suggesting that the increase of $\mathrm{N} / \mathrm{O}$ with $\mathrm{N} / \mathrm{H}$ is due to the increase of $\mathrm{N}$, without modifying the oxygen abundance through the $\mathrm{ON}$ cycle. Theoretical predictions of N/O vs. $\mathrm{N} / \mathrm{H}$ for different masses of the progenitor stars and metallicity (Groenewegen \& de Jong 1994 for the LMC metallicity, and Marigo 2001 for $Z=0.004$ ) are also shown. The theoretical predictions of Groenewegen $\&$ de Jong (1994), shown by the solid line in Fig. 10, and of Marigo (2001), the dashed line, do not extend to the low metallicities of Sextans A and Sextans B. Because of it, we can only say that models, in particular those of Marigo (2001) predict the deviation from the linear relationship of Henry (1989) that the observations of PNe in Sextans B and in the Magellanic Clouds suggest at the lowest metallicities. From the figure there is no 
Table 6. Total chemical abundances of Sextans A and Sextans B PNe computed with both ICF and CLOUDY methods. We also report the unweigthed average of the two results. Metal abundances are expressed in $12+\log (\mathrm{X} / \mathrm{H})$.

\begin{tabular}{|c|c|c|c|c|c|c|}
\hline Name & $\mathrm{He} / \mathrm{H}$ & $\mathrm{N} / \mathrm{H}$ & $\mathrm{O} / \mathrm{H}$ & $\mathrm{Ne} / \mathrm{H}$ & $\mathrm{S} / \mathrm{H}$ & $\mathrm{Ar} / \mathrm{H}$ \\
\hline \multicolumn{7}{|l|}{ SexA-PN } \\
\hline ICF & $0.084 \pm 0.016$ & $8.35 \pm 0.2$ & $8.1 \pm 0.1$ & $6.6 \pm 0.2$ & $5.8 \pm 0.1$ & $5.2 \pm 0.4$ \\
\hline CLOUDY & $0.095 \pm 0.02$ & $8.4 \pm 0.2$ & $8.0 \pm 0.1$ & $6.7 \pm 0.2$ & $5.9 \pm 0.2$ & $5.2 \pm 0.2$ \\
\hline Average & $0.09 \pm 0.02$ & $8.4 \pm 0.2$ & $8.0 \pm 0.1$ & $6.7 \pm 0.2$ & $5.9 \pm 0.2$ & $5.2 \pm 0.3$ \\
\hline \multicolumn{7}{|l|}{ SexB-PN1 } \\
\hline ICF & $0.099 \pm 0.015$ & $6.7:$ & $8.0 \pm 0.15$ & $7.2 \pm 0.3$ & - & - \\
\hline CLOUDY & $0.10 \pm 0.02$ & 6.6: & $8.1 \pm 0.3$ & $7.3 \pm 0.3$ & - & - \\
\hline Average & $0.10 \pm 0.02$ & 6.6: & $8.1 \pm 0.2$ & $7.3 \pm 0.3$ & - & - \\
\hline \multicolumn{7}{|l|}{ SexB-PN2 } \\
\hline ICF & $0.096 \pm 0.016$ & $6.7:$ & $7.7 \pm 0.3$ & $6.8 \pm 0.2$ & - & - \\
\hline CLOUDY & $0.11 \pm 0.02$ & 6.6: & $8.0 \pm 0.2$ & $7.0 \pm 0.3$ & - & - \\
\hline Average & $0.10 \pm 0.02$ & 6.6: & $7.8 \pm 0.2$ & $6.9 \pm 0.3$ & - & - \\
\hline \multicolumn{7}{|l|}{ SexB-PN3 } \\
\hline ICF & $0.076 \pm 0.016$ & $>5.5 \dagger$ & $7.5 \pm 0.3$ & $6.9 \pm 0.3$ & $>4.9 \dagger$ & $5.7 \pm 0.2$ \\
\hline CLOUDY & $0.09 \pm 0.02$ & $6.5 \pm 0.3$ & $7.6 \pm 0.2$ & $7.1 \pm 0.2$ & 5.2: & $5.5 \pm 0.2$ \\
\hline Average & $0.08 \pm 0.02$ & $6.5 \pm 0.3$ & $7.5 \pm 0.2$ & $7.0 \pm 0.2$ & 5.2: & $5.6 \pm 0.2$ \\
\hline \multicolumn{7}{|l|}{ SexB-PN4 } \\
\hline ICF & $0.095 \pm 0.014$ & $6.8 \pm 0.3$ & $8.3 \pm 0.1$ & $7.4 \pm 0.1$ & $5.3 \pm 0.2$ & - \\
\hline CLOUDY & $0.09 \pm 0.02$ & $6.3 \pm 0.3$ & $8.2 \pm 0.2$ & $7.4 \pm 0.2$ & $5.2 \pm 0.2$ & 5.1: \\
\hline Average & $0.09 \pm 0.02$ & $6.5 \pm 0.3$ & $8.2 \pm 0.2$ & $7.4 \pm 0.2$ & $5.2 \pm 0.2$ & 5.1: \\
\hline \multicolumn{7}{|l|}{ SexB-PN5 } \\
\hline ICF & $0.13 \pm 0.02$ & $7.5 \pm 0.3$ & $8.2 \pm 0.1$ & $7.6 \pm 0.2$ & $5.4 \pm 0.2$ & - \\
\hline CLOUDY & $0.13 \pm 0.02$ & $7.0 \pm 0.3$ & $8.2 \pm 0.2$ & $7.6 \pm 0.2$ & $5.7 \pm 0.2$ & - \\
\hline Average & $0.13 \pm 0.02$ & $7.3 \pm 0.3$ & $8.2 \pm 0.1$ & $7.6 \pm 0.2$ & $5.5 \pm 0.2$ & - \\
\hline Average Sextans B & $0.10 \pm 0.02$ & $6.8 \pm 0.6$ & $8.0 \pm 0.3$ & $7.3 \pm 0.3$ & $5.3 \pm 0.4$ & - \\
\hline
\end{tabular}

Table 7. $T_{\star}$ and $L_{\star}$ of the PNe in Sextans A and Sextans B as derived with the modelling with CLOUDY.

\begin{tabular}{lllc}
\hline \hline Galaxy & Name & $T_{\star}(\mathrm{K})$ & $\log \left(L_{\star} / L_{\odot}\right)$ \\
\hline Sextans A & PN & 194000 & 3.9 \\
Sextans B & PN1 & 61000 & 3.5 \\
& PN2 & 78000 & 3.3 \\
& PN3 & 72000 & 3.5 \\
& PN4 & 65000 & 3.2 \\
& PN5 & 90000 & 3.6 \\
\hline
\end{tabular}

indication of oxygen enrichment for PNe with low metallicity and very low mass progenitors $\left(M<1.3 M_{\odot}\right)$, as also predicted by theoretical models of Marigo (2001). The location in the diagram of the Sextans A PN is a clear indication of a large nitrogen production, as expected from its higher mass progenitor, while oxygen production is not evident from this diagram.

Figure 11 presents the relation between $\mathrm{O} / \mathrm{H}$ and $\mathrm{Ne} / \mathrm{H}$. It is usually believed that the progenitors of PNe do not produce significant amount of oxygen and neon during their lifetime. In fact, the abundances of these two elements seem to vary in lockstep, where the slope of the log-log relation is very close to unity (cf. Henry 1989). This behaviour is essentially the same for $\mathrm{H}$ II regions and it seems independent of the host galaxy. In Fig. 11 the solid line is the fit built with a sample of $157 \mathrm{PNe}$ in the Galaxy, LMC, SMC and M 31 taken from Henry (1989). The dotted lines are placed at a distance of $\pm 1 \sigma$ from the fit, and the dashed lines at $\pm 3 \sigma$, where $\sigma=0.1$ dex. The PNe and the H II regions of Sextans A and Sextans B appear to follow the "universal" relation. 
Table 8. The chemical abundances of H II regions in Sextans A. Where the electron temperature is measured, values derived with both the CLOUDY and the ICF methods are indicated. In the remaining cases, marked with a “*”, only the determinations done with CLOUDY are listed. In computing the average abundances, upper limits of individual determinations are not considered.

\begin{tabular}{|c|c|c|c|c|c|c|}
\hline Name & $\mathrm{He} / \mathrm{H}$ & $\mathrm{N} / \mathrm{H}$ & $\mathrm{O} / \mathrm{H}$ & $\mathrm{Ne} / \mathrm{H}$ & $\mathrm{S} / \mathrm{H}$ & $\mathrm{Ar} / \mathrm{H}$ \\
\hline \multicolumn{7}{|l|}{ HKS2* } \\
\hline CLOUDY & $<0.05$ & $6.1 \pm 0.3$ & $7.7 \pm 0.2$ & $6.7 \pm 0.2$ & $6.1 \pm 0.2$ & $5.3 \pm 0.3$ \\
\hline \multicolumn{7}{|l|}{ HKS3 } \\
\hline ICF & $0.05 \pm 0.01$ & $6.2 \pm 0.1$ & $7.5 \pm 0.1$ & $6.9 \pm 0.2$ & $6.0 \pm 0.1$ & $5.3 \pm 0.1$ \\
\hline CLOUDY & $0.05 \pm 0.02$ & $6.3 \pm 0.3$ & $7.6 \pm 0.2$ & $6.6 \pm 0.3$ & $6.1 \pm 0.2$ & $5.3 \pm 0.3$ \\
\hline Average & $0.05 \pm 0.02$ & $6.2 \pm 0.2$ & $7.5 \pm 0.1$ & $6.7 \pm 0.2$ & $6.0 \pm 0.2$ & $5.3 \pm 0.2$ \\
\hline \multicolumn{7}{|l|}{ HKS7 } \\
\hline ICF & $0.08 \pm 0.01$ & $5.9 \pm 0.2$ & $7.2 \pm 0.2$ & $6.4 \pm 0.2$ & $5.9 \pm 0.1$ & $5.1 \pm 0.3$ \\
\hline CLOUDY & $0.07 \pm 0.02$ & $6.2 \pm 0.3$ & $7.6 \pm 0.2$ & $6.6 \pm 0.3$ & $6.0 \pm 0.2$ & $5.4 \pm 0.3$ \\
\hline Average & $0.07 \pm 0.02$ & $6.1 \pm 0.3$ & $7.4 \pm 0.2$ & $6.5 \pm 0.2$ & $6.0 \pm 0.2$ & $5.3 \pm 0.3$ \\
\hline \multicolumn{7}{|l|}{ HKS9 } \\
\hline ICF & $0.03 \pm 0.01$ & $6.1 \pm 0.2$ & $7.2 \pm 0.2$ & $6.5 \pm 0.2$ & $5.8 \pm 0.1$ & $5.2 \pm 0.3$ \\
\hline CLOUDY & $0.03 \pm 0.02$ & $6.5 \pm 0.3$ & $7.3 \pm 0.2$ & $6.5 \pm 0.3$ & $5.8 \pm 0.2$ & $5.1 \pm 0.3$ \\
\hline Average & $0.03 \pm 0.02$ & $6.3 \pm 0.2$ & $7.2 \pm 0.1$ & $6.5 \pm 0.2$ & $5.8 \pm 0.2$ & $5.1 \pm 0.3$ \\
\hline \multicolumn{7}{|l|}{ HKS14 } \\
\hline ICF & & $6.3 \pm 0.2$ & $7.7 \pm 0.1$ & $7.0 \pm 0.2$ & $5.9 \pm 0.2$ & $5.2 \pm 0.3$ \\
\hline CLOUDY & $<0.05$ & $6.3 \pm 0.3$ & $7.9 \pm 0.2$ & $7.0 \pm 0.3$ & $6.1 \pm 0.2$ & $5.2 \pm 0.3$ \\
\hline Average & $<0.05$ & $6.3 \pm 0.3$ & $7.8 \pm 0.1$ & $7.0 \pm 0.2$ & $6.0 \pm 0.2$ & $5.2 \pm 0.3$ \\
\hline \multicolumn{7}{|l|}{ HKS16* } \\
\hline CLOUDY & $<0.05$ & $6.0 \pm 0.3$ & $7.7 \pm 0.2$ & $6.4 \pm 0.2$ & $6.0 \pm 0.2$ & $5.5 \pm 0.3$ \\
\hline \multicolumn{7}{|l|}{ HKS19* } \\
\hline CLOUDY & $<0.05$ & $6.2 \pm 0.3$ & $7.6 \pm 0.2$ & $6.9 \pm 0.2$ & $6.2 \pm 0.2$ & $5.0 \pm 0.3$ \\
\hline \multicolumn{7}{|l|}{ HKS23* } \\
\hline CLOUDY & $<0.05$ & $6.2 \pm 0.3$ & $7.7 \pm 0.2$ & $6.7 \pm 0.2$ & $5.8 \pm 0.2$ & $5.0 \pm 0.3$ \\
\hline \multicolumn{7}{|l|}{ HKS24* } \\
\hline CLOUDY & $<0.05$ & $6.3 \pm 0.3$ & $7.6 \pm 0.2$ & $7.1 \pm 0.2$ & $6.3 \pm 0.2$ & $4.7 \pm 0.3$ \\
\hline Average values & $0.05 \pm 0.02$ & $6.2 \pm 0.1$ & $7.6 \pm 0.2$ & $6.8 \pm 0.3$ & $6.0 \pm 0.2$ & $5.2 \pm 0.2$ \\
\hline
\end{tabular}

\subsection{The distribution of chemical abundances within the galaxy}

As star formation in dwarf irregular galaxies is thought to be episodic, and the solid-body nature of the rotation inhibits efficient mixing, it is plausible that there are large abundance variations in different regions of a galaxy (Skillman et al. 1989). However, in several dwarf and low-mass galaxies it has been found that the $\mathrm{O} / \mathrm{H}$ abundance appears surprisingly homogeneous. There are several explanations which can be considered, as described, for example, by Kobulnicky (1998). This is true also in the case of Sextans A and Sextans B. The abundance of their best measured element, oxygen, has a low scatter in $\mathrm{H}$ II regions: the average value is $7.6 \pm 0.2$ for Sextans $\mathrm{A}$ and
$7.8 \pm 0.2$ for Sextans B, being, therefore, consistent within errors with an uniform distribution.

Another aspect to be considered is the so called "abundance gap", which is the difference between the abundances of H II regions and PNe (cf. Richer et al. 1997). Generally H II regions have larger $\mathrm{O} / \mathrm{H}$ than $\mathrm{PNe}$, because they belong to a younger population than the PN progenitors, which are not considered to produce large amount of oxygen, unless under "special" circumstances (see Sect. 4). From the models of Richer et al. (1997), this gap is expected to be as large as 0.2 or 0.3 dex, but it is a function of the metallicity and of the chemical history of the galaxy. Both in Sextans A and Sextans B this gap does not seem to be present (for Sextans A, we considered $\mathrm{Ne} / \mathrm{H}$ instead of the oxygen abundance, which might be enhanced during the 
Table 9. The same as in Table 8, but for Sextans B.

\begin{tabular}{lllllll}
\hline \hline Name & $\mathrm{He} / \mathrm{H}$ & $\mathrm{N} / \mathrm{H}$ & $\mathrm{O} / \mathrm{H}$ & $\mathrm{Ne} / \mathrm{H}$ & $\mathrm{S} / \mathrm{H}$ & $\mathrm{Ar} / \mathrm{H}$ \\
\hline SHK1 & & & & & & \\
ICF & - & $6.0 \pm 0.2$ & $7.5 \pm 0.1$ & $6.6 \pm 0.2$ & $5.9 \pm 0.2$ & $5.3 \pm 0.2$ \\
CLOUDY & $<0.05$ & $6.1 \pm 0.3$ & $7.6 \pm 0.1$ & $6.5 \pm 0.3$ & $6.0 \pm 0.2$ & $5.3 \pm 0.3$ \\
Average & $<0.05$ & $6.0 \pm 0.2$ & $7.6 \pm 0.1$ & $6.5 \pm 0.2$ & $5.9 \pm 0.4$ & $5.3 \pm 0.3$ \\
\hline SHK2 & & & & & & \\
ICF & $0.12 \pm 0.02$ & $6.2 \pm 0.2$ & $7.5 \pm 0.1$ & $7.0 \pm 0.2$ & $6.2 \pm 0.2$ & $5.8 \pm 0.2$ \\
CLOUDY & $0.11 \pm 0.02$ & $6.4 \pm 0.3$ & $7.7 \pm 0.1$ & $7.0 \pm 0.3$ & $6.2 \pm 0.2$ & $5.8 \pm 0.3$ \\
Average & $0.11 \pm 0.02$ & $6.3 \pm 0.2$ & $7.6 \pm 0.1$ & $7.0 \pm 0.2$ & $6.2 \pm 0.2$ & $5.8 \pm 0.3$ \\
\hline SHK3* & & & & & & \\
CLOUDY & $<0.05$ & $6.7 \pm 0.3$ & $8.0 \pm 0.2$ & - & $6.7 \pm 0.2$ & - \\
\hline SHK5 & & & & & & \\
ICF & $0.04 \pm 0.01$ & $6.4 \pm 0.2$ & $7.7 \pm 0.1$ & $7.0 \pm 0.2$ & $6.0 \pm 0.2$ & $4.9 \pm 0.2$ \\
CLOUDY & $0.04 \pm 0.02$ & $6.3 \pm 0.3$ & $7.7 \pm 0.1$ & $7.0 \pm 0.3$ & $6.1 \pm 0.2$ & $5.0 \pm 0.3$ \\
Average & $0.04 \pm 0.02$ & $6.3 \pm 0.2$ & $7.7 \pm 0.1$ & $7.0 \pm 0.2$ & $6.0 \pm 0.2$ & $5.0 \pm 0.3$ \\
\hline SHK7* & & & & & & \\
CLOUDY & $<0.05$ & $6.4 \pm 0.3$ & $7.7 \pm 0.2$ & - & $6.1 \pm 0.2$ & $5.5 \pm 0.3$ \\
\hline SHK6* & & & & & & \\
CLOUDY & $<0.05$ & $6.6 \pm 0.3$ & $7.8 \pm 0.2$ & - & $6.6 \pm 0.2$ & - \\
\hline SHK10* & & & & & & \\
CLOUDY & $<0.05$ & $6.4 \pm 0.3$ & $8.1 \pm 0.2$ & $7.0 \pm 0.2$ & $6.0 \pm 0.2$ & $5.5 \pm 0.3$ \\
\hline H IIH* & & & & & & \\
CLOUDY & $0.05 \pm 0.02$ & $6.1 \pm 0.3$ & $7.8 \pm 0.2$ & $6.9 \pm 0.3$ & $6.0 \pm 0.2$ & $5.0 \pm 0.3$ \\
\hline Average values & $0.07 \pm 0.04$ & $6.4 \pm 0.2$ & $7.8 \pm 0.2$ & $7.0 \pm 0.2$ & $6.1 \pm 0.5$ & $5.5 \pm 0.4$ \\
\hline & & & & & & \\
\hline
\end{tabular}

evolution of the progenitor of the PN, see discussion in Sect. 4). For Sextans A, its PN has a young, relatively massive progenitor and thus it is not surprising that it has abundances similar to those of $\mathrm{H}$ II regions.

For Sextans B, the old ages of the PNe progenitors estimated in Sect. 3.3, together with their homogeneous abundances, would indicate a very low chemical enrichment of the galaxy during a long period of time till the present. Determining with more accuracy the length of this period of low chemical enrichment (5-10 Gyrs?) is prevented by the significant uncertainties and approximations done in deriving the ages of the PN progenitors.

The average $\mathrm{O} / \mathrm{H}$ abundances of HII regions in Sextans A and Sextans B are slightly different $(7.6 \pm 0.2$ vs. and $7.8 \pm 0.2$, respectively), but they are within the errors and computed dispersion, and therefore no clear indication of a different chemical history can be drawn. In fact, as Sextans A and Sextans B have approximately the same V-luminosity and the same morphological type, considering the well known linear relationship between the metallicity and the luminosity of galaxies (see Pilyugin 2001), they are expected to have the same $\mathrm{O} / \mathrm{H}$, within the dispersion of the empirical relationship. So the similar $\mathrm{O} / \mathrm{H}$ abundances, in spite of quite different SFHs, seem to confirm that galaxies with a similar luminosity have similar metallicity and that the luminosity-metallicity relationship represents the ability of a given galaxy to keep the products of its own evolution rather than its ability to produce metals (Larson 1974).

\section{The peculiar PN of Sextans $A$}

The PN of Sextans A shows significant N/H and O/H overabundances with respect to the $\mathrm{H}$ II regions. $\mathrm{N} / \mathrm{H}$ is generally enhanced in $\mathrm{PNe}$ by nucleosynthesis processes in the progenitor star. The large amount of $\mathrm{N} / \mathrm{H}$ in this $\mathrm{PN}$ can be explained, as for type I PN in the Galaxy (Corradi \& Schwarz 1995), by a relatively high mass progenitor which produces and dredgesup nitrogen. On the other hand, the abundances of neon, sulphur, and argon, which are not expected to be varied by nucleosynthesis, are similar, within errors, to the average values of $\mathrm{H}$ II regions (or slightly lower, consistently with the fact the PN belongs to a slightly older stellar population). 


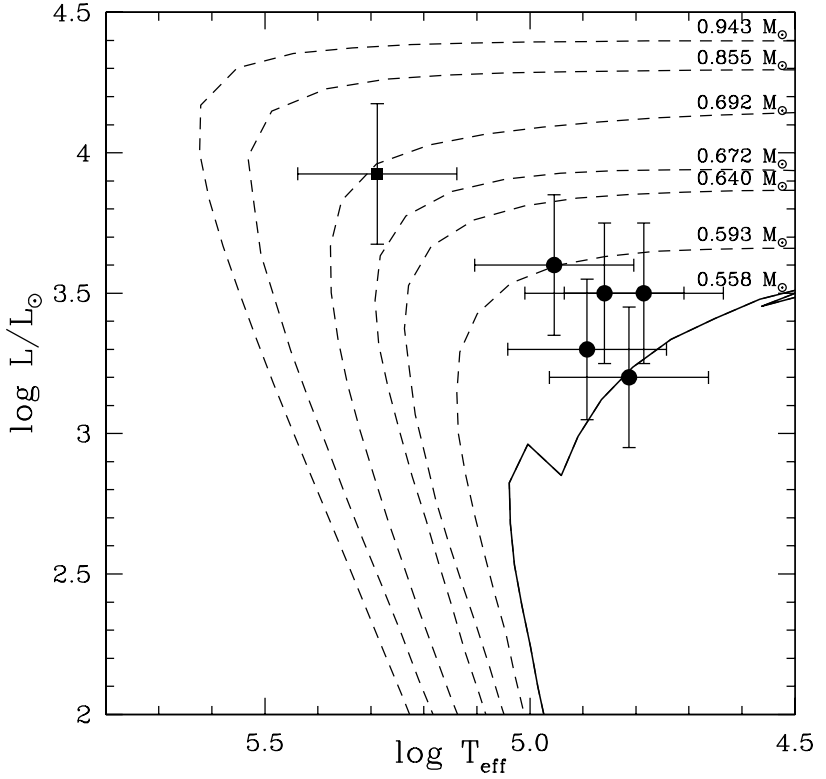

Fig. 8. H-burning (dashed lines) and He-burning (continuous line) evolutionary tracks for metallicity $Z=0.004$ from Vassiliadis \& Wood (1994). Final mass of the central star are quoted close to the corresponding track. Large symbols (circles) correspond to Sextans B PNe and the small one (square) indicates the Sextans A PN.

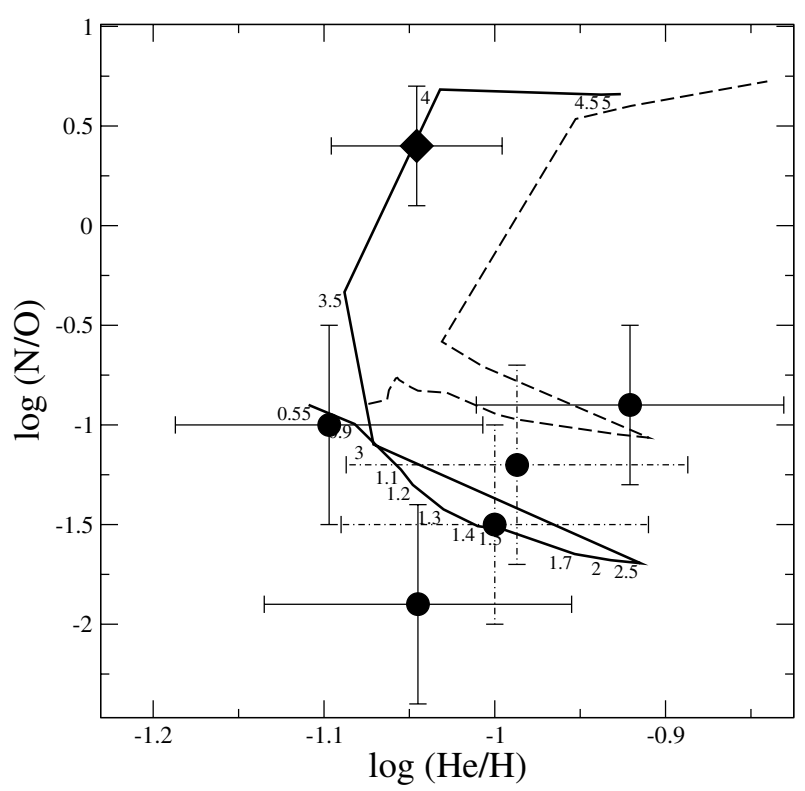

Fig. 9. Relationship between $\mathrm{He} / \mathrm{H}$ and N/O. The solid line is the stellar model for the progenitor by Marigo (2004) for $Z=0.001$ and the dashed line for $Z=0.004$. Values of the initial masses are indicated. Full circles correspond to Sextans B PNe and the diamond is the Sextans A PN. PNe with uncertain N/H determination are marked with dot-dashed error bars.

The overabundance of oxygen is more difficult to understand. As noted in Sect. 3.6, the $\mathrm{O} / \mathrm{H}$ abundance of the $\mathrm{H}$ II regions studied in Sextans A has a low dispersion, indicating that there is today an homogeneous distribution of elements in the galaxy. These data, however, do not allow us to exclude the existence of a metallicity gradient through Sextans A, because the $\mathrm{H}$ II regions are located roughly in a ring, and there are no

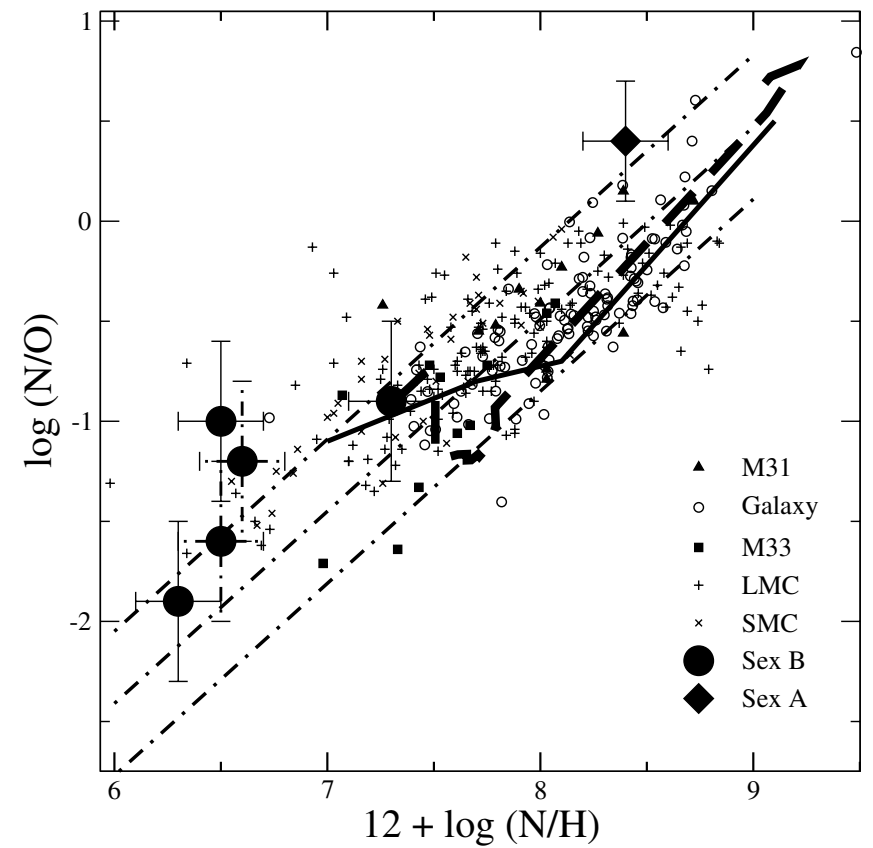

Fig. 10. Relationship between N/O and N/H for PNe. The central dotdashed line is the fit from Henry (1990) using a sample of PNe of the Galaxy, LMC, SMC and M31. The external dot-dashed lines are placed at a distance of $\pm 3 \sigma$ from the fit, where $\sigma=0.1 \mathrm{dex}$. The solid line is the model for LMC by Groenewegen \& de Jong (1994) built for the LMC metallicity and several initial masses. The top-right point of the curve corresponds to a model with an initial mass of $8.5 M_{\odot}$, the first change of slope with $2 M_{\odot}$, and the last point (bottom-left) is for $0.93 M_{\odot}$. The dashed line is the model for $Z=0.004$ and initial masses from 0.8 (left) to 5 (right) $M_{\odot}$ by Marigo (2001). Observed abundances are from: Galaxy (Perinotto et al. 2004a); LMC and SMC (Leisy et al., in prep.); M 31 (Jacoby \& Ciardullo 1999); M 33 (Magrini et al. 2004); Sextans A and Sextans B (this work). PNe of Sextans B with uncertain $\mathrm{N} / \mathrm{H}$ determination are marked with dot-dashed error bars.

data for the central region where the PN is located (it should also be remembered that the PN progenitor may have been migrated from its original place of birth). Existing information from the literature appears contradictory, as while the three Atype supergiant stars located over a length of $0.8 \mathrm{kpc}$ studied by Kaufer et al. (2004) have uniform Fe/H abundances, other stars show a larger r.m.s scatter in metallicity at all ages (up to 0.4 dex for intermediate mass stars, Dolphin et al. 2003, or for red giant branch stars, Grebel et al. 2003). However, the hypothesis that the progenitor of the PN has formed in a region of Sextans A with locally enhanced metallicity seems to be ruled out (or at least weakened) by the fact that only $\mathrm{O} / \mathrm{H}$ is significantly higher than in HII regions, while the abundance of other elements which do not vary during stellar evolution of the PN progenitor, like sulphur, argon, and neon, are all very similar to those of the $\mathrm{H}$ II regions.

An alternative, interesting explanation is that $\mathrm{O} / \mathrm{H}$ surface enrichment occurred during the evolution of the PN progenitor. This was first suggested by Leisy et al. (2002) for several $\mathrm{PNe}$ in the Magellanic Clouds and interpreted as a consequence of a dredge-up that depends on the metallicity of the PN progenitor and enriches of oxygen its surface. Indeed, contrary to 


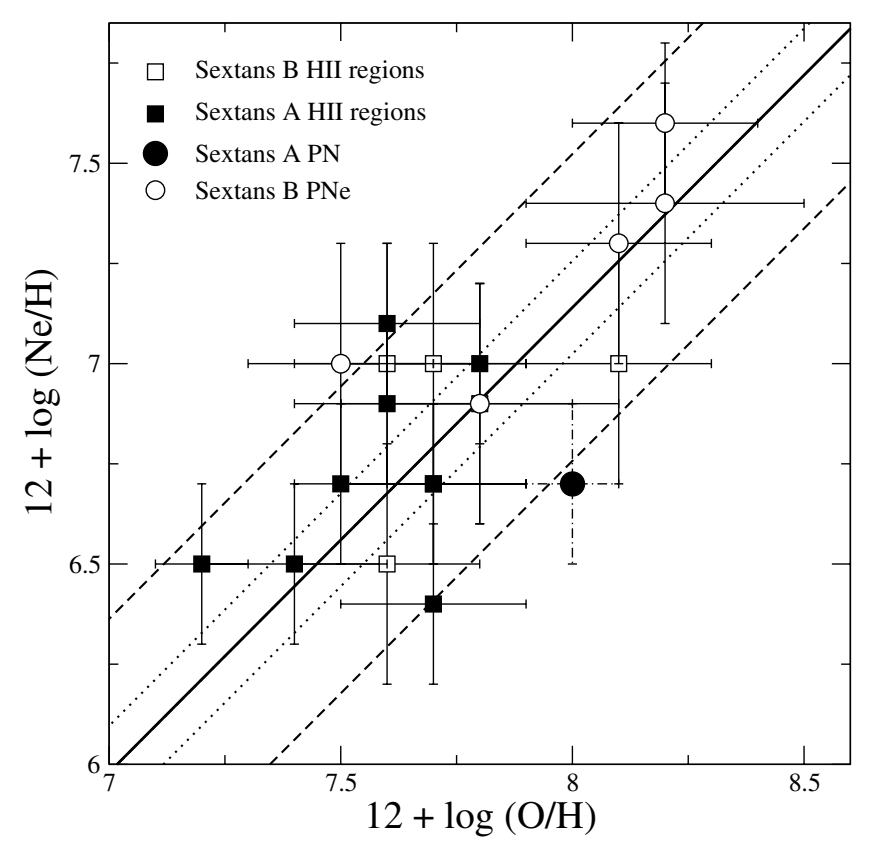

Fig. 11. Relationship between $\mathrm{Ne} / \mathrm{H}$ and $\mathrm{O} / \mathrm{H}$. The solid line is the fit built by Henry (1989). The dotted lines are placed at a distance of $\pm 1 \sigma$ from the fit, and the dashed lines at $\pm 3 \sigma$, where $\sigma=0.1 \mathrm{dex}$ as indicated by Henry 1989. Only H II regions with $T_{\mathrm{e}}$ measured are reported.

"classical" stellar evolution theories, recent semi-analytical models do predict $\mathrm{O}$ enrichment by core processed material brought to the surface by the third dredge-up (cf. Marigo 2001; Herwig 2004). In the model of Marigo (2001), the O production depends on several parameters: the metallicity (increasing at low metallicities), the mixing length, and the number of dredge-up episodes (which also increases at low metallicities because of the larger duration of the AGB phase). At a given progenitor mass of $2 M_{\odot}$, Marigo (2001) found an increase of the total oxygen stellar yield of a factor 10, when lowering the initial metallicity from solar to $Z=0.004$. The oxygen enrichment becomes important only for progenitors with $M>1.3 M_{\odot}$. In a recent paper, Herwig (2004) computed intermediate mass (from 2 to $6 M_{\odot}$ ) stellar evolution tracks from the main sequence to the tip of the AGB for starts with extremely low metallicities, namely $Z=0.0001$. These models predict that oxygen is enhanced in the envelopes of low metallicity AGB stars by the third dredge-up. The new model of Marigo (2004, private communication), built for an initial metallicity $Z=0.001$ (that of Sextans A), also predicts that the third dredge-up produces some oxygen enrichment, as seen by the slight decreasing trend of N/O with increasing $\mathrm{He} / \mathrm{H}$ in Fig. 9. In particular, for a PN with a progenitor of about $3.5 M_{\odot}$ and an initial metallicity of $Z=0.001$, Marigo (2004) found that hot bottom burning and third dredge-up are present. The production of oxygen is essentially due to the third dredge-up and it amounts to about 0.4 dex, in (surprisingly good) agreement with the difference found between the PN and the $\mathrm{H}$ II regions of Sextans A. Concluding, our observations of the PN in Sextans A support the possibility that a significant oxygen surface enrichment can occur during the evolution of highest mass PN progenitors born in low metallicity environments.
This potentially important result is nevertheless based on the only known example of a PN with a high mass progenitor belonging to such a low metallicity galaxy as Sextans A. Confirming this results is an important objective of future observational research in the field.

\section{Summary}

In this paper we presented a homogeneous spectroscopic study of ionized nebulae ( $\mathrm{H}$ II regions and $\mathrm{PNe}$ ) representative of populations of different ages, in the two low-metallicity, dwarf irregular galaxies Sextans A and Sextans B. Our main results are:

i) we have computed, via ICF and CLOUDY modeling, helium, nitrogen, oxygen, neon, argon, and sulphur abundances both in $\mathrm{H}$ II regions and PNe. In particularly, the element with the most accurate determination, oxygen, has the same average value, within the errors, in the PNe and $\mathrm{H}$ II regions of Sextans B: $12+\log (\mathrm{O} / \mathrm{H})=8.0 \pm 0.3$ and $7.8 \pm 0.2$, respectively, indicating a very low metal enrichment during a significant fraction of the galaxy lifetime. For the $\mathrm{H}$ II regions of Sextans A we obtained $12+\log (\mathrm{O} / \mathrm{H})=7.6 \pm 0.2$, whereas it is 0.4 dex larger for the only PN known there;

ii) the dispersion of $\mathrm{O} / \mathrm{H}$ in Sextans $\mathrm{A}$ and Sextans B H II regions has a rms scatter of 0.2 dex, which is consistent, within the errors, with an uniform distribution;

iii) the oxygen overabundance of the PN in Sextans A with respect to HII regions might indicate an efficient third dredge-up for massive, low-metallicity PN progenitors as predicted by models of Marigo (2001, 2004).

Acknowledgements. We are grateful to P. Marigo for the help given in the interpretation of our results using the preliminary results of her new AGB models for low metallicity stars. We thank an anonymous referee for comments and suggestions which have improved the paper.

\section{References}

Ambartsumian, V. A. 1932, Poulkovo Obs. Circ., 4, 8

Aparicio, A., \& Rodríguez-Ulloa, J. A. 1992, A\&A, 260, 77

Charbonnel, C., Meynet, G., Maeder, A., Schaller, G., \& Schaerer, D. 1993, A\&AS, 101, 415

Charbonnel, C., Meynet, G., Maeder, A., \& Schaerer, D. 1996, A\&AS, 115,339

Charbonnel, C., Dåppen, W., Schaerer, D., et al. 1999, A\&AS, 135, 405

Corradi, R. L. M., \& Magrini, L. 2004, Proc. ESO-workshop on Planetary Nebulae beyond the Milky Way, to appear

Corradi, R. L. M., \& Schwarz, H. 1995, A\&A, 293, 871

Corradi, R. L. M., Perinotto, M., Schwarz, H. E., \& Claeskens, J.-F. 1997, A\&A, 322, 975

Dolphin, A. E., Saha, A., Skillman, E. D., et al. 2003, AJ, 126, 187

Dopita, M. A. 1996, Proc. of the Second Recontres du Vietnam ed. J. Tran Thanh Van, L. M. Celnikier, Hua Chon Trung, \& S. Vauclair (Éditions Frontieres), 295

Exter, K. M., Barlow, M. J., \& Walton, N. A. 2004, MNRAS, 349, 1291

Ferland, G. J., Korista, K. T., Verner, D. A., et al. 1998, PASP, 110, 761 
Filippenko, A. V. 1982, PASP, 94, 715

Grebel, E. K. 2000, in Star Formation from the Small to the Large Scale, ed. F. Favata, A. A. Kaas, \& A. Wilson (Noordwijk: ESA), SP-445, 87

Grebel, E. K., Gallagher, J. S. III, \& Harbeck, D. 2003, AJ, 125, 1926 Grevesse, N., \& Sauval, A. J. 1998, SSRv, 85, 161

Groenewegen, M. A. T., \& de Jong, T. 1994, A\&A, 282, 127

Gurzadyan, G. A. 1988, Ap\&SS, 149, 343

Henry, R. B. C. 1989, MNRAS, 241, 453

Henry, R. B. C. 1990, ApJ, 356, 229

Herwig, F. 2000, ApJ, 605, 425

Herwig, F. 2004, ApJS, 155, 651

Hodge, P. W. 1974, ApJS, 27, 113

Hodge, P., Kennicutt, R. C., \& Strobel, N. 1994, PASP, 106, 765

Huchtmeier, W. K., Karachentsev, I. D., \& Karachentseva, V. E. 2003, A\&A, 401, 483

Hunter, D. A., Hawley, W. N., \& Gallagher, J. S. 1993, AJ, 106, 1797

Kaufer, A., Venn, K. A., Tolstoy, E., Pinte, C., \& Kudritzki, R.-P. 2004, AJ, 127, 2723

Kingsburgh, R. L., \& Barlow, M. J. 1994, MNRAS, 271, 257

Kniazev, A. Y., Grebel, E. K., Pustilnik, S. A., Pramskij, A. G., \& Zucker, D. B. 2005 [arXiv: astro-ph/0502562]

Kobulnicky, H. A. 1998, ASPC, 147, 108

Jacoby, G. H., \& Lesser, M. P. 1981, AJ, 86, 185

Jacoby, G. H., \& Ciardullo, R. 1999, ApJ, 515, 169

Larson, R. B. 1974, MNRAS, 169, 229

Leisy, P., Francois, P., \& Dennefeld, M. 2002, RMAA, 12, 140

Magrini, L., Corradi, R. L. M., Walton, N. A., et al. 2002, A\&A, 386, 869

Magrini, L., Corradi, R. L. M., Greimel, R., et al. 2003, A\&A, 407, 51

Magrini, L., Perinotto, M., Mampaso, A., \& Corradi, R. L. M. 2004, A\&A, 426, 779
Marigo, P. 2001, A\&A, 370, 194

Marigo, P., Bressan, A., \& Chiosi, C. 1996, A\&A, 313, 545

Mateo, M. L. 1998, ARA\&A, 36, 435

Mathis, J. S. 1990, ARA\&A, 28, 37

McKenna, F. C., Keenan, F. P., Kaler, J. B., et al. 1996, PASP, 108, 610

Moles, M., Aparicio, A., \& Masegosa, J. 1990, A\&A, 228, 310

Pagel, B. E. J. 1998, in Nucleosynthesis and chemical evolution of galaxies (Cambridge Univ. Press), 289

Perinotto, M., \& Corradi, R. L. M. 1998, A\&A, 332, 721

Perinotto, M., Morbidelli, L., \& Scatarzi, A. 2004a, MNRAS, 349, 793

Perinotto, M., Magrini, L., Mampaso, A., \& Corradi, R. L. M. 2004b, Proc. ESO-workshop, Planetary Nebulae beyond the Milky Way, to appear

Pilyugin, L. S. 2000, A\&A, 362, 325

Pilyugin, L. S. 2001, A\&A, 374, 412

Richer, M. P., \& McCall, M. L. 1995, ApJ, 445, 642

Richer, M. P., \& McCall, M. L. 2004, Proc. ESO-workshop, Planetary Nebulae beyond the Milky Way, to appear

Richer, M. G., McCall, M. L., \& Arimoto, N. 1997, A\&AS, 122, 215

Skillman, E. D., Kennicutt, R. C., \& Hodge, P. W. 1989, ApJ, 347, 875

Stanghellini, L., \& Kaler, J. B. 1989, ApJ, 343, 811

Stasinska, G. 2002, in Proc. of Ionized Gaseous Nebulae, ed. W. J. Henney, J. Franco, M. Martos, \& M. Peña, RMAA, 12, 62

Strobel, N. V., Hodge, P., \& Kennicutt, R. C. Jr. 1991, ApJ, 383, 148

Tosi, M., Greggio, L., Marconi, G., \& Forcardi, P. 1991, AJ, 102, 951

van den Bergh, S. 2000, in The Galaxies of the Local Group (Cambridge University Press), vdB00

Vassiliadis, E., \& Wood, P. R. 1994, ApJS, 92, 125 